\title{
Studies in Ion Source Development for Application in Heavy Ion Fusion
}

\author{
Project Report \\ Master of Science - Nuclear Engineering \\ University of California, Berkeley
}

Jonathan G. Kapica

May 2004 


\section{Contents}

1. INTRODUCTION TO PROJECT 1

1.1 Objective and Requirements for HIF 1

1.2 STS 50 and Aluminosilicate Sources 2

1.3 STS 100 and Plasma Sources 4

2. ALUMINO-SILICATE SOURCE DC PULSE EXPERIMENTS 5

2.1 Introduction to Experiment 5

2.2 DC Power Supply Design and Construction 5

$\begin{array}{lll}2.3 & \text { Potassium Alumino-silicate Source } & 7\end{array}$

$\begin{array}{lll}2.4 & \text { Cesium Alumino-silicate Source } & 14\end{array}$

$\begin{array}{ll}2.5 \text { Conclusion from Experiment } & 16\end{array}$

3. TIME OF FLIGHT MEASUREMENTS OF DC BEAM 17

3.1 Motivation and Basic Principle of Experiment 17

$\begin{array}{lll}3.2 & \text { Experimental Setups } & 17\end{array}$

3.3 Experimental Results 19

3.4 Development of Model and Comparison to Measured Data 25

3.5 Contaminated Cs Source 29

3.6 Conclusions from Experiment 32

4. ALUMINO-SILICATE SOURCE LIFETIME EXPERIMENTS 33

4.1 Setup and method of measurement 33

4.2 Experimental Results 33

$\begin{array}{lll}4.3 & \text { Conclusions } & 38\end{array}$

5. PLASMA SOURCE ENERGY ANALYZER EXPERIMENT 39

5.1 Introduction 39

5.2 Principles and Theory 40

5.2.1 Optics of a Thin Lens 40

5.2.2 Energy Analyzer Principles 42

5.2.3 Charge Exchange in the Diode Gap 45 
5.3 Experiment Setup 48

5.3.1 Source Setup 48

5.3.2 Energy Analyzer Setup 48

5.3.3 High Voltage Pulse $\quad 50$

5.4 Single Beam Results 51

5.4.1 Adjustments to Instrument 51

5.4.2 Argon Gas Pressure Variations 53

5.4.3 Scanning of Electrode-Voltage 53

5.4.4 Asymmetric Electrode-Voltage Test 55

5.5 Combined Beam Results 55

5.6 Energy Spread Due to Charge Exchange 57

5.7 Conclusions $\quad 59$

6. REFERENCES AND ACKNOWLEDGMENTS 60 


\section{Introduction to Project}

\subsection{Objective and Requirements for HIF}

The overall purpose of these experiments is to contribute to the development of ion injector technology in order to produce a driver for use in a heavy-ion-fusion (HIF) power generating facility. The overall beam requirements for HIF are quite demanding; a short list of the constraints is the following:

- Low cost (a large portion of overall cost will come from the beam system)

- Bright, low emittance beam

- Total beam energy 5MJ

- Spot size 3mm (radius)

- Pulse Duration 10ns

- Current on target 40kA

- Repetition Rate $5 \mathrm{~Hz}$

- Standoff from target $5 \mathrm{~m}$

- Transverse Temp $<1 \mathrm{keV}$

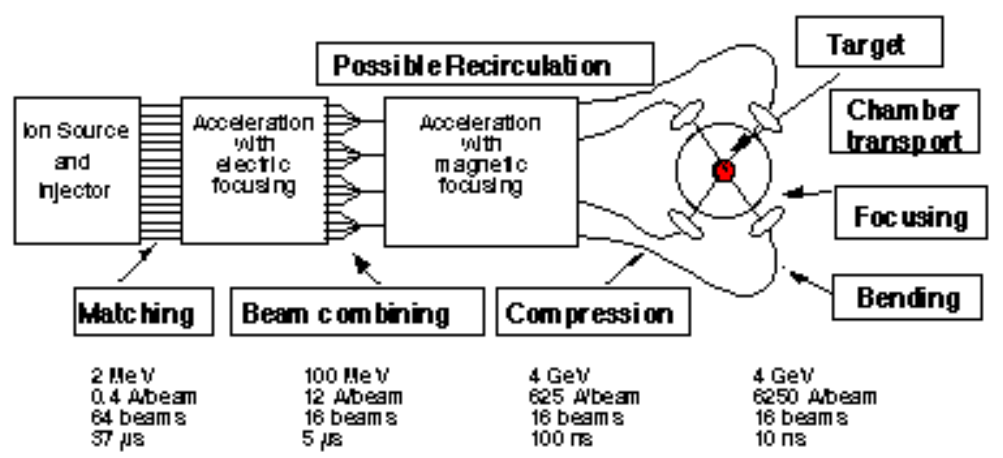

Figure 1.1. Layout of basic linear accelerator used for generating the required beam energy on target for HIF. (Figure taken from UCB Tutorial on Heavy-Ion Fusion).

The reasons for employing ion beams in inertial fusion systems become obvious when the repetition rate required is considered. While laser drivers are useful in producing a proof-of-concept, they will be incapable of application in power generation. Consequently attempts in the U.S. to achieve a power generating system make use of linear ion accelerators. It is apparent that the accelerator system requires the highest 
quality input as obtainable. Therefore injector design is an essential portion of the entire inertial fusion system.

At Lawrence Berkeley and Lawrence Livermore National Laboratories experiments are being conducted using two injector formats. For this project I have conducted a series of studies using both. The next two sections provide a brief description of the sources used for my experiments.

\subsection{STS 50 and Alumino-Silicate Sources}

The STS 50 test stand located at LBNL and shown in figure 1.2 consists of a small vacuum chamber containing a themionic ion gun, diagnostics such as a Faraday cup, slit scanners, neutral-detector, and a time-of-flight measuring apparatus.

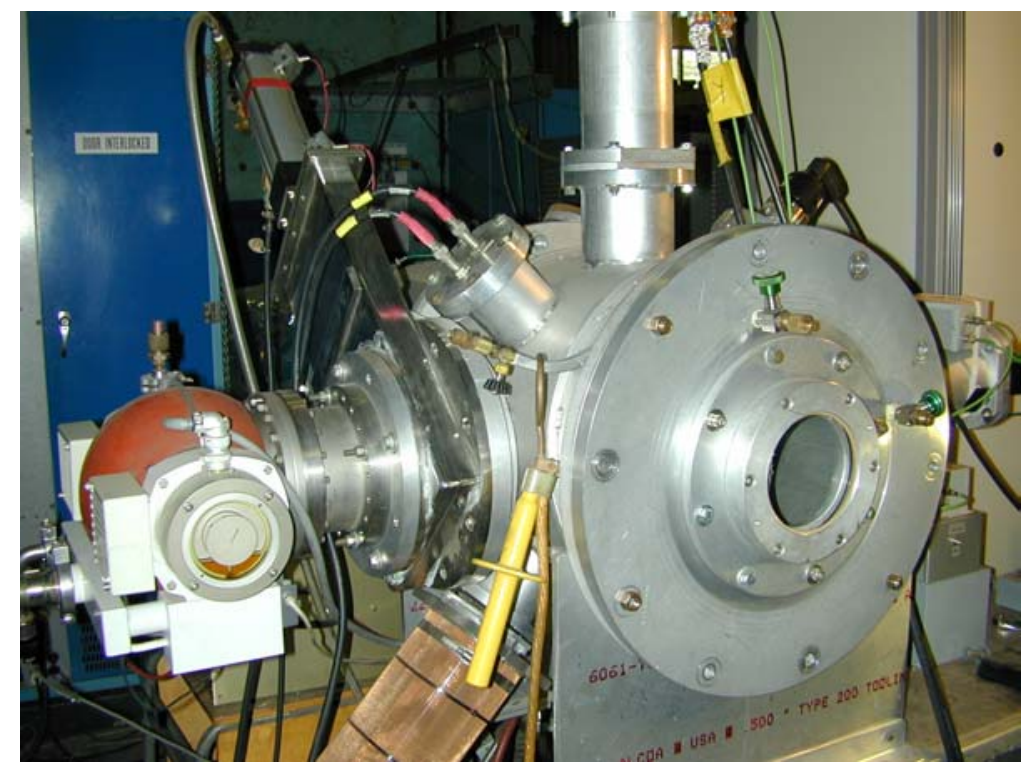

Figure 1.2. STS50 ion source test stand at LBNL.

Vacuum is maintained by combining a roughing pump and turbo pump. Pressure is monitored with a cold cathode and for most experiments operates in the $1 \times 10^{-7} \mathrm{~T}$ range. The ion gun holds a $1 / 4$ inch diameter tungsten source (coated with alumino-silicate) in a heat shielded cup containing tungsten heating filaments shown in figure 1.3. Power is 
supplied to filaments by an isolation transformer. Figure 1.4 shows a side view of the assembled gun displaying the extraction gap. The gap distance is adjustable and the source fits into a Pierce electrode. The Pierce (not seen in picture) provides a shaped acceleration potential to produce a laminar beam with uniform current density.

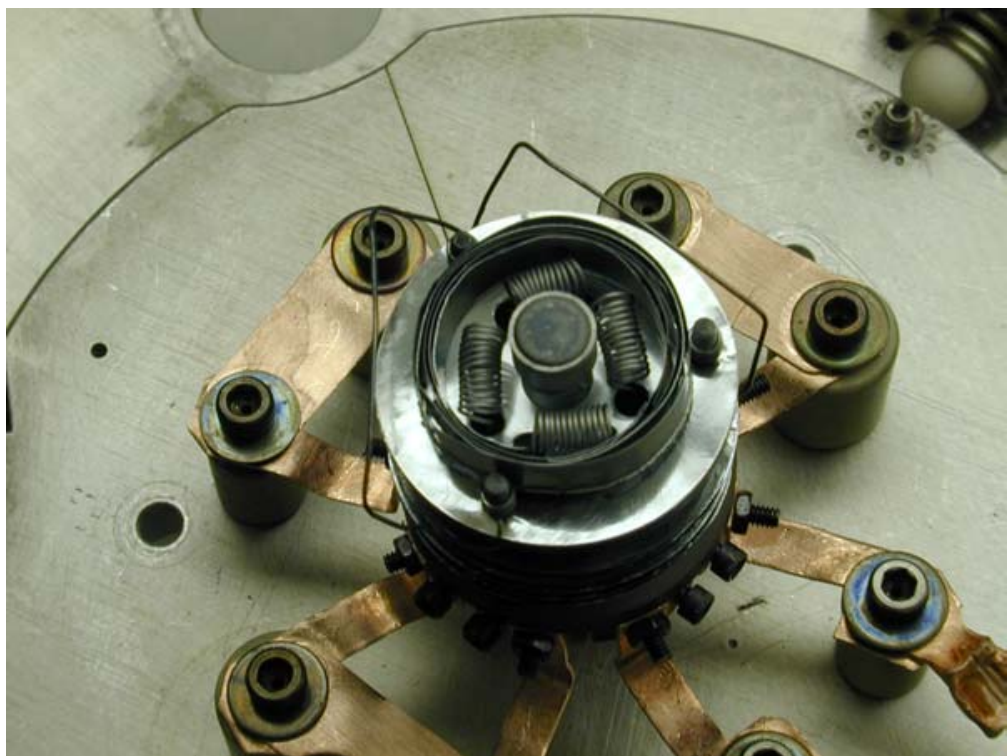

Figure 1.3. $1 / 4$ inch source used in STS50. Source shown with heating system.

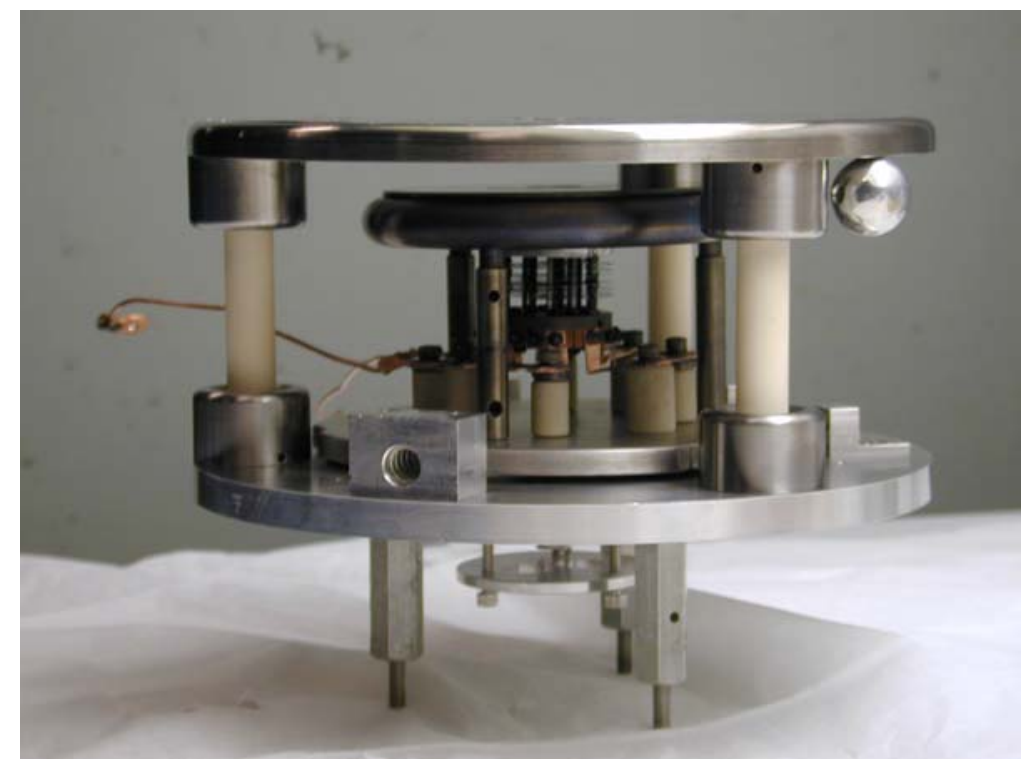

Figure 1.4. Ion gun assembly used in STS 50.

Extraction pulses are generated with two different systems for these experiments. The initial testing was conducted with a bi-polar $\pm 35 \mathrm{kV}$ thyratron-triggered pulse 
forming network. For most of the experiments, however, a long duration low voltage, $\pm 2.5 \mathrm{kV}$, supply was used. This supply is discussed further in section 2. Extensive discussion regarding the development and fabrication of the alumino-silicate source is contained in the two references Chacon-Glocher, E., 2002, and Baca D., et al. 2003. Alumino-silicate, surface ionization sources provide reliable and relatively long-life performance and have been a preferred choice for experimentation due to their simplicity and low cost.

\subsection{STS 100 and Plasma Sources}

STS 100 at LLNL is the ion source used for the energy spread experiments. It operates with an RF driven plasma source. The source is configured with a $26 \mathrm{~cm}$ inner diameter cylindrical plasma chamber, multi-cusp permanent magnet confinement, 11 $\mathrm{MHz} / 20 \mu \mathrm{s}$ RF pulse, through a 2 turn $11 \mathrm{~cm}$ antenna. Ar gas is fed through a time controlled puffer valve. The multi-beam array consists of a 61 hole grid in a hexagonal pattern. The beam forming plate contains $2.2 \mathrm{~mm}$ Pierce cones, the extraction plate has matching $4.0 \mathrm{~mm}$ holes and the gap between the plates is $1.6 \mathrm{~cm}$, capable of holding $80 \mathrm{kV}$. The pulser is capable of $100 \mathrm{kV}, 20 \mu$ s pulses, produced from a spark-gap triggered system. The primary diagnostic used for this project was an electrostatic energy analyzer, which is discussed at length in chapter 5 . 


\section{Alumino-Silicate Source DC Pulse Experiments}

\subsection{Introduction to Experiment}

The following series of experiments have been conducted to better understand the performance of an alumino-silicate source for applications in heavy-ion and magnetic fusion systems. Of interest is the diffusion of ions from the source and the resulting current from applying a long DC extraction potential. Additionally, from long extraction periods, the characteristics of the source over the lifetime can be studied. The experiments have been performed at LBNL on the STS50 Ion Source Test Stand, using a $1 / 4 "$ diameter $\left(\mathrm{A}=0.317 \mathrm{~cm}^{2}\right)$ tungsten substrate source.

\subsection{Power Supply Design and Construction}

In order to accomplish this series of long pulse-length experiments a power supply and switching system was constructed. To achieve the desired extraction pulse the system was required to provide long pulses up to $24 \mathrm{hrs}$, supply steady pulse with constant voltage regardless of beam loading, switch on and off easily with signal generator input, and output + and $-2.5 \mathrm{kV}$. The system was built using two high-voltage insulated gate bipolar transistors (IGBT). A functional schematic of the system is shown in figure 2.1. The IGBT serves as a switch to apply the power supply output to the respective electrode on the ion gun. Each IGBT is controlled by a driver that applies the appropriate bias voltages to turn the device on or off. To isolate high-voltage, the drivers are connected to the signal generator through analog to optical converters. A sample of the system output is shown in figure 2.2 . 


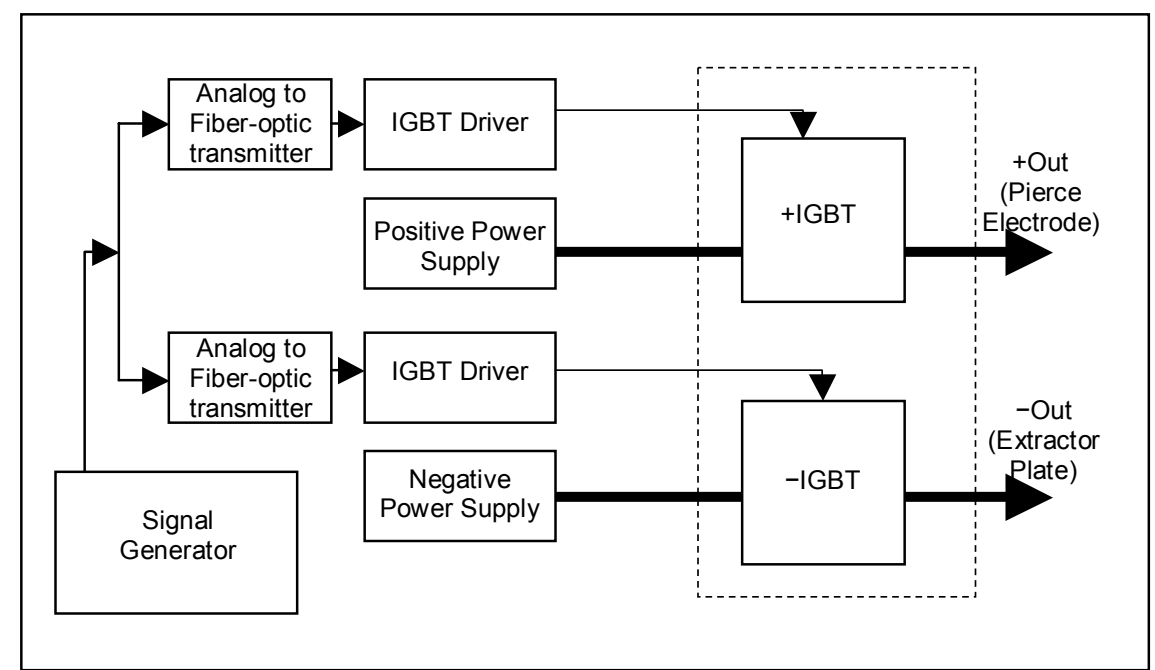

Figure 2.1. DC power supply setup.

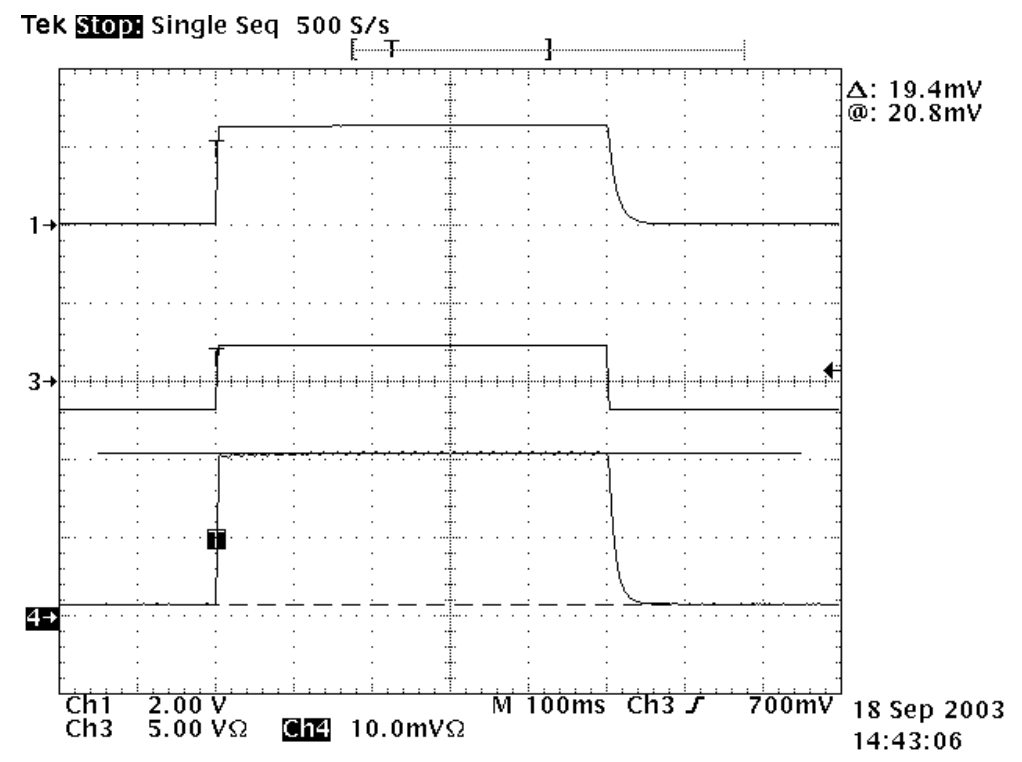

Figure 2.2. Oscilloscope image showing three traces: 1 - positive $2.5 \mathrm{kV}$ extraction voltage, 3 - signal generator pulse $5 \mathrm{~V}, 4$ - faraday cup signal $19.4 \mathrm{mV}$. All shown on a $100 \mathrm{~ms} / \mathrm{div}$ scale.

The following series of tests was run to verify proper operation of the system. A sample of results is plotted in figure 2.3. The unusual shape of the decay and the properties of the steady state value will be investigated in the following and future experiments. 


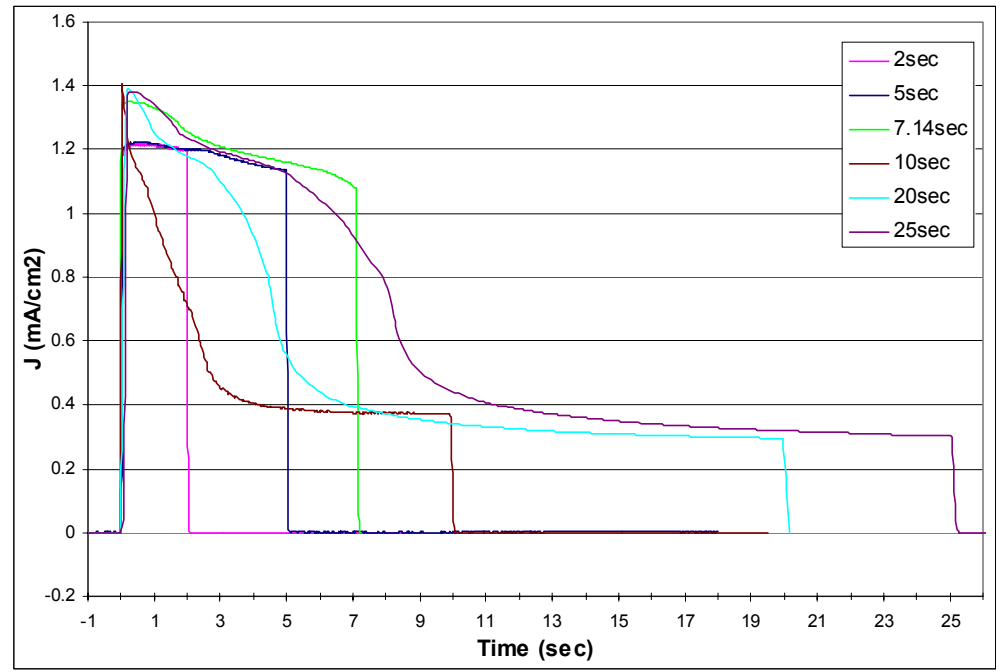

Figure 2.3. Beam current density at various pulse lengths at $5 \mathrm{kV}$ temp $1100 \mathrm{C}$.

\subsection{Potassium Alumino-Silicate Source}

This series of experiments was conducted with a Potassium alumino-silicate source. Originally the STS50 test stand was equipped with a 50kV short $(20 \mu \mathrm{s})$ duration Marx pulse system. To gain an understanding of this system and the operating characteristics of the ion gun at these low extraction voltages, beam current was compared to previous results. Shown in figure 2.4 is a comparison of the current density from the $5 \mathrm{kV}$ DC setup to previously recorded data on the $50 \mathrm{kV}$ setup. The current varies significantly for the duration of the long pulse. In order to compare DC to fast pulse results, the DC signal is measured a two points, the initial peak and the steady-state value at the tail. Initially beam current peaks at a value that is clearly space-charge limited and is shown in the graph of figure 2.4. As expected, the peak current density scales proportional to $\mathrm{V}^{3 / 2}$. 


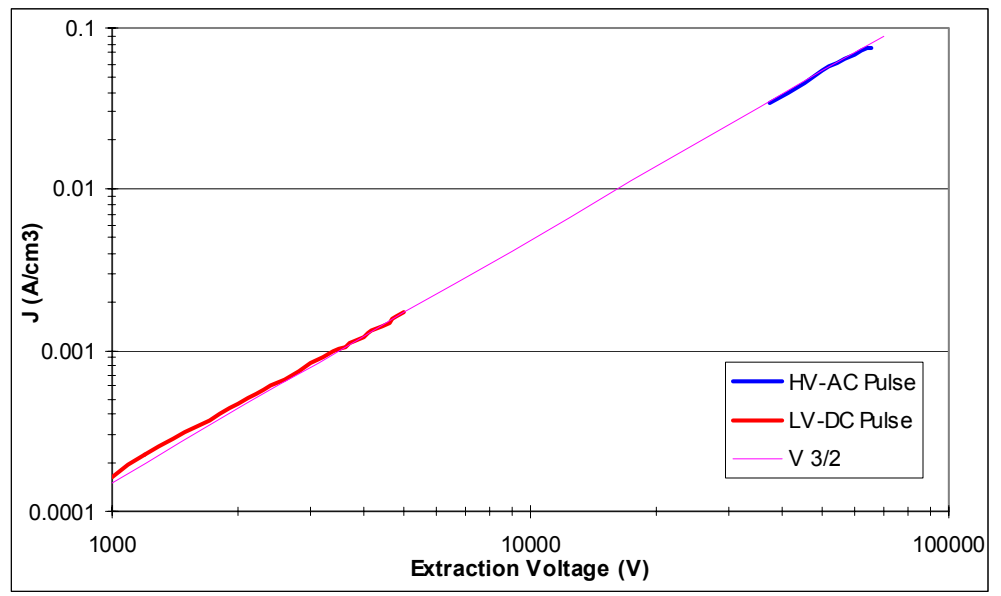

Figure 2.4. J vs. V curves for high voltage AC pulses $(20 \mu \mathrm{s})$ and peak values from the low voltage DC pulses $(500 \mathrm{~ms})$, temperature $1090^{\circ} \mathrm{C}$.

For the following test, the affect of source temperature on the peak and steady state values of current density is determined using the following setup:

- Chamber pressure $3.4 \times 10^{-7} \mathrm{~T}$ for entire experiment

- Suppressor voltage $-800 \mathrm{~V}$

- Faraday cup directly connected to scope internal $50 \Omega$ load (spark-gap used for scope protection) no bias voltage applied.

- Data taken in order of ascending temperature

- Temperatures measured with pyrometer before and after data acquisition

Starting with source temperature at $1050^{\circ} \mathrm{C}$, recorded the $\mathrm{FC}$ signal at five voltages 1 to $5 \mathrm{kV}$ in $1 \mathrm{kV}$ steps using $5 \mathrm{~ms}$ pulse length. The same set of data was taken at 1105 and $1175^{\circ} \mathrm{C}$ and the results, plotted on a log scale in figure 2.5 . Once again peak values were used.

From the graph of figure 2.5 it can be seen that at $1105^{\circ} \mathrm{C}$ and $1175^{\circ} \mathrm{C}$ current appears to be space-charge limited, while at $1050^{\circ} \mathrm{C}$ it is emission limited. 


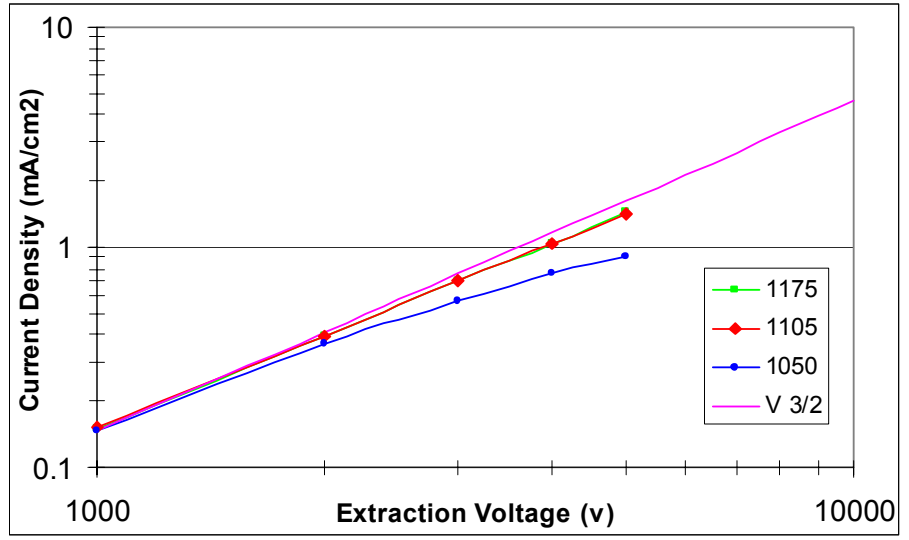

Figure 2.5. Current density vs. Extraction voltage $(1-5 \mathrm{kV})$ at three temperatures $\left({ }^{\circ} \mathrm{C}\right)$.

After the data for the $1050^{\circ} \mathrm{C}$ case in figure 2.5 was obtained, a few long pulses $(10 \mathrm{sec})$ were run and caused an increase in the cup current for the same voltage. To better understand this difference the current-density vs. voltage data was retaken and is shown in figure 2.6. The temperature was measured before and after each data run and was constant within the accuracy of the pyrometer, however this method only measures the temperature of the source surface. The long pulse apparently causes some type of conditioning making the source operate closer to the space-charge limit. This effect has been noted in many other cases and appears to be a characteristic property of the alumino-silicate sources. I believe current flow from the source produces heat from $i^{2} R$ losses, and more evenly heats the source internally. Once the source "conditions" it continues to operate at the higher current until it is shutdown or depleted. 


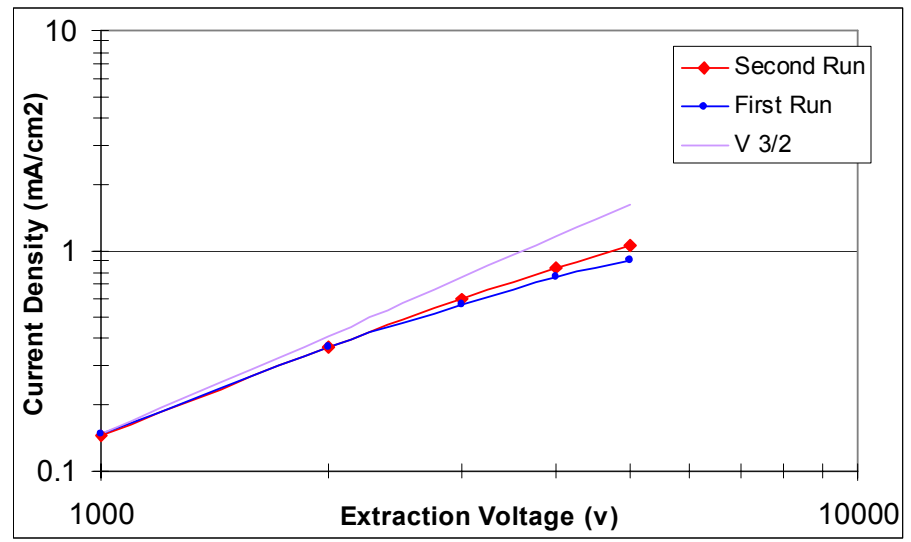

Figure 2.6. Density vs. Voltage at same temp after several long pulses.

The following set of figures $(2.7-2.9)$ is shown to display the long pulse time characteristics of the source and the effects of source temperature. In figure 2.7 the $1050^{\circ} \mathrm{C}$ case droops more while $1105^{\circ} \mathrm{C}$ and $1175^{\circ} \mathrm{C}$ cases hold fairly constant. This shows a difference in ion diffusion rate from the source as a function of temperature. Clearly operating the source at higher temperatures maintains a more uniform current density for the duration of the pulse. When the length of the extraction is pushed out to $20 \mathrm{sec}$ the resulting behavior becomes interesting and is shown in figure 2.8. Here the $1050^{\circ} \mathrm{C}$ case drops off significantly and displays a double decay rate possibly indicating two different diffusion rates.

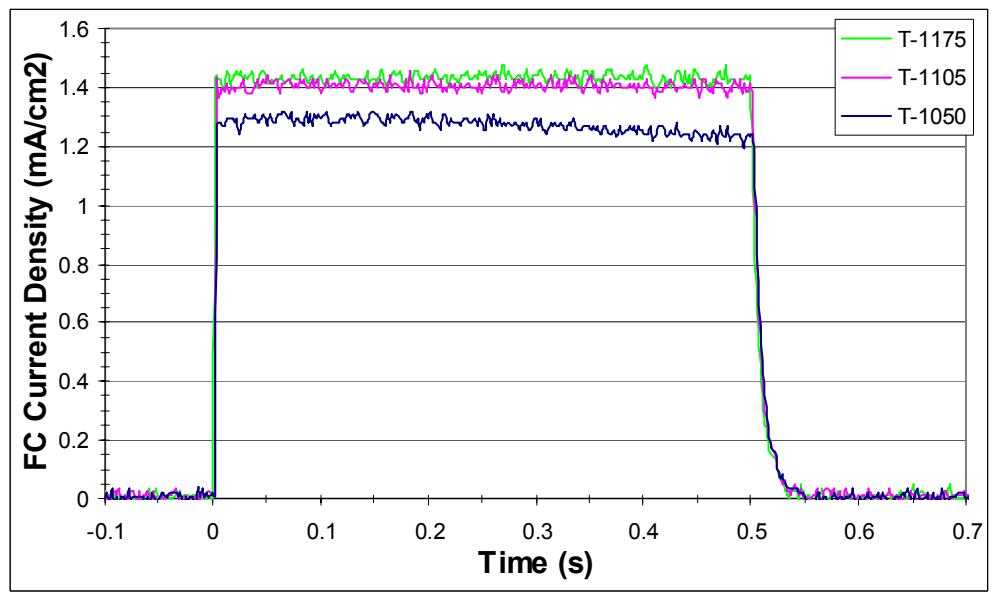

Figure 2.7. $500 \mathrm{~ms}$ pulse at $5 \mathrm{kV}$ for different source temperatures. 


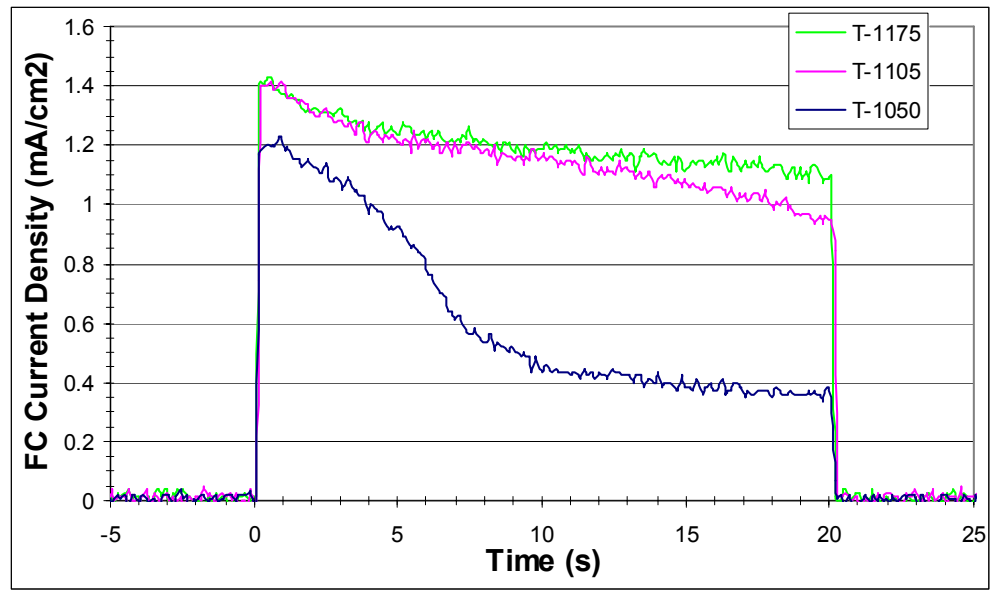

Figure 2.8. 20 second pulse at $5 \mathrm{kV}$.

The dramatic change in diffusion rate is clearly seen in figure 2.9 for all temperatures. All three cases decay to a steady state level. The steady state level appears to be a function of temperature.

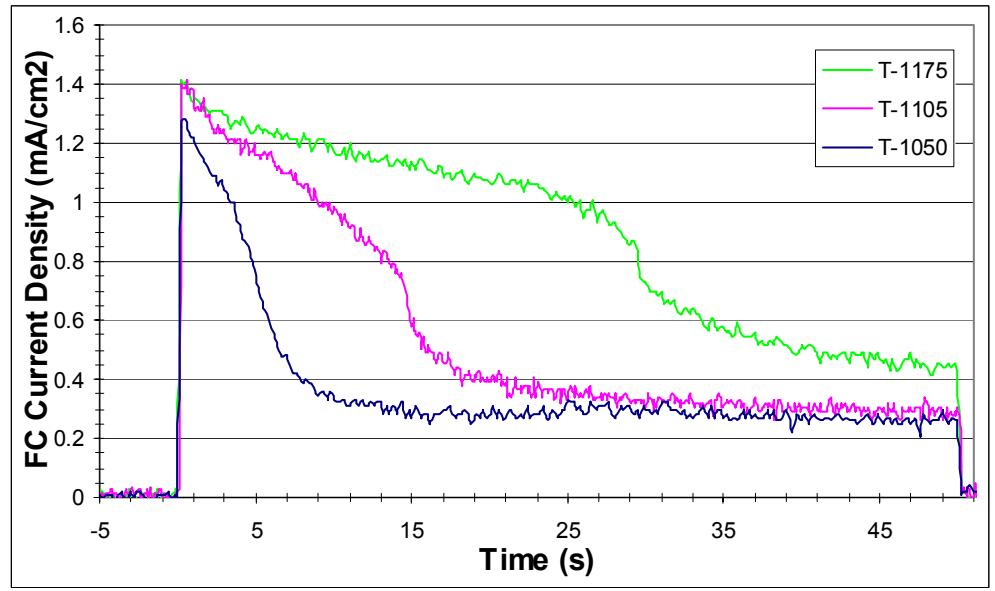

Figure 2.9. 50 second pulse at $5 \mathrm{kV}$.

Figure 2.10 shows the behavior of the $1175^{\circ} \mathrm{C}$ case past the 50 second point, at which it slowly tapers off to a value slightly higher than the two lower temperature cases. 


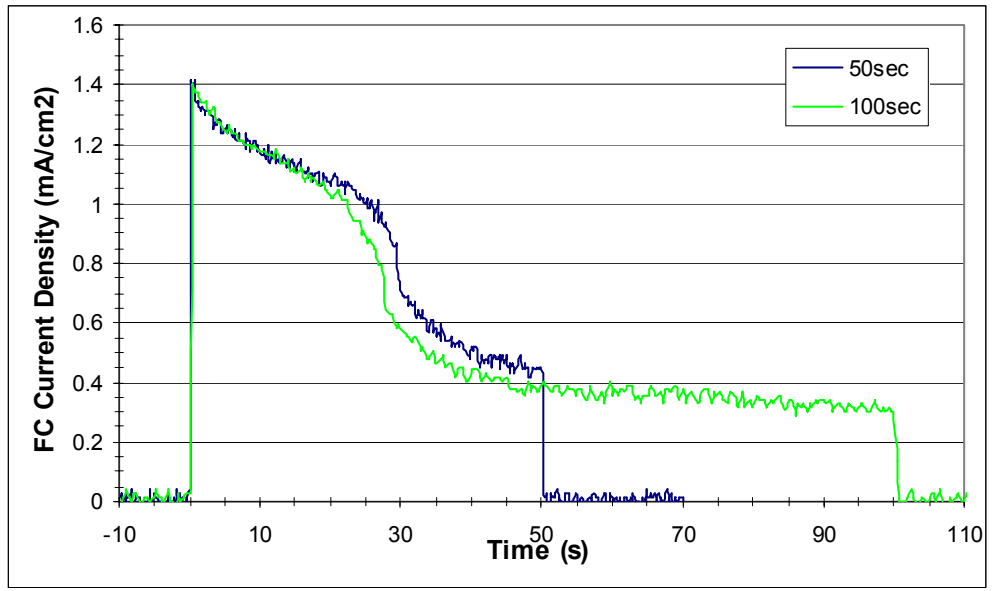

Figure 2.10. 100 and 50 second pulses $1175^{\circ} \mathrm{C}$ at $5 \mathrm{kV}$.

In an attempt to explain the decay rate the curves were fitted using the following equations:

$$
\begin{gathered}
J=J_{o} \exp \left(-\frac{\Delta t}{\tau}\right) \\
\tau=\tau_{o} \exp \left(\frac{Q}{T}\right)
\end{gathered}
$$

$T$ is temperature in units of energy and $Q$ is desorption energy of the species. Two decay rates of figure 2.11 may be result of two ion species. Species one depletes as function of $\tau_{1}$ and the second species depletes at a rate proportional to $\tau_{2}$. From the values of these rates it can be seen that $\tau_{1}$ is half $\tau_{2}$. It appears from the above equations the only variable in $\tau$ that changes is $Q$ which is a property of the species. 


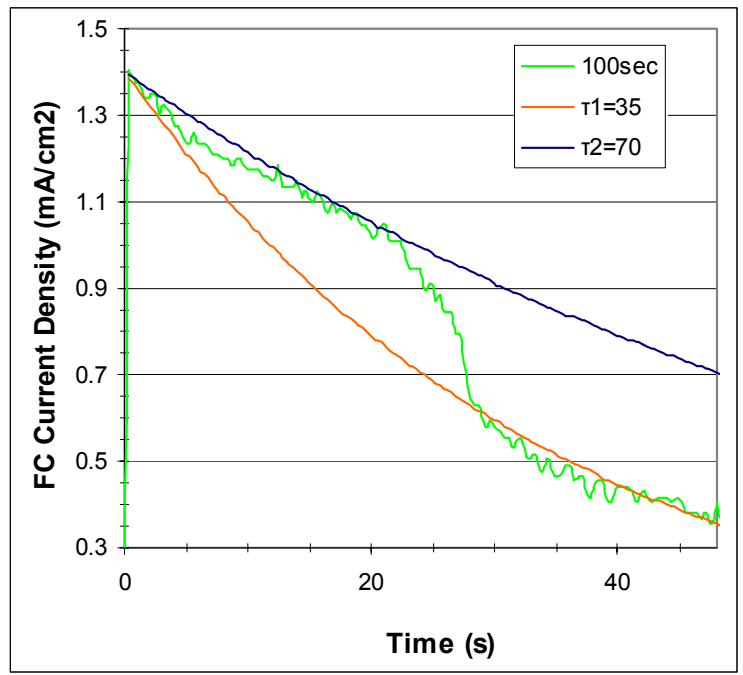

Figure 2.11. Close-up of figure 2.10 showing decay rates with fitted exponential curves.

After about 50 seconds for this source, beam current appears to decay to a fairly stable steady-state value. During quick pulses (on the order of $\mu$ s) with adequate rest between shots, the source has an opportunity to replenish the ion density in the extraction gap to a space-charge limit. However, during the DC condition, ions are continuously cleared from the gap and at this point the emission of ions from the source should be equal to beam current. The emission limited results shown in figure 2.12 were obtained by measuring the average beam current of the last $50 \mathrm{~ms}$ of $50 \mathrm{sec}$ long pulses.

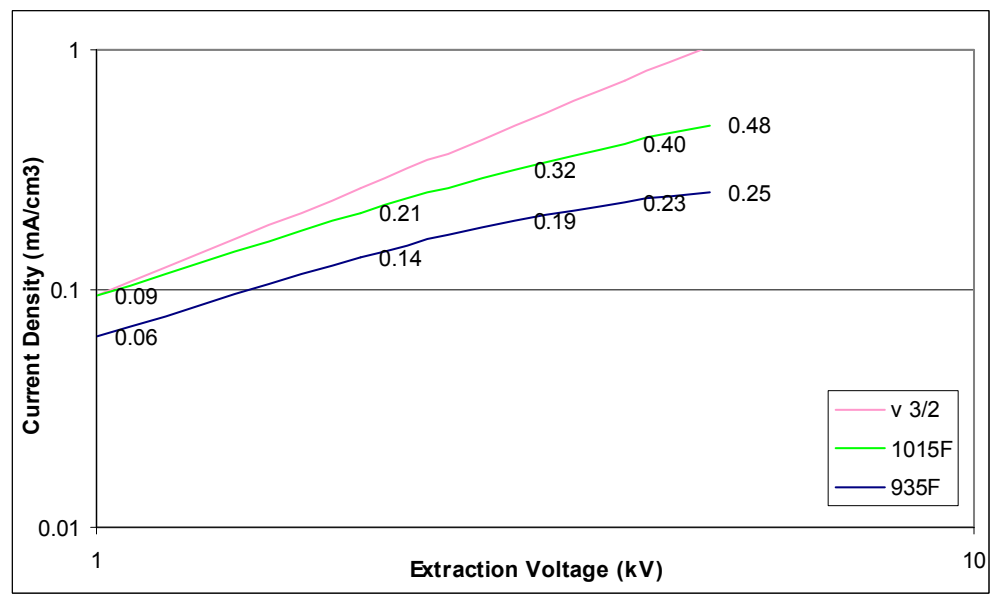

Figure 2.12. J vs. Voltage during the steady state value of the last $50 \mathrm{~ms}$ of a $50 \mathrm{sec}$ pulse. 


\subsection{Cesium Alumino-Silicate Source}

The following results were obtained using a 1/4" Cesium Alumino-silicate source.

Figure 2.13 shows the operating regions of $500 \mathrm{~ms}$ pulses at varied temperatures. For the 1 to $5 \mathrm{kV}$ results, the peak values were seen during the first $4 \mathrm{~ms}$ of the pulse, and the tail values are taken from an average of the last $100 \mathrm{~ms}$ of the pulse. The 20 to $50 \mathrm{kV}$ values were obtained from $20 \mu$ s pulses generated using the STS50 high-voltage Marx PS. The peak DC values and the AC results follow the space-charge limit, while the steady-state values are clearly emission limited.

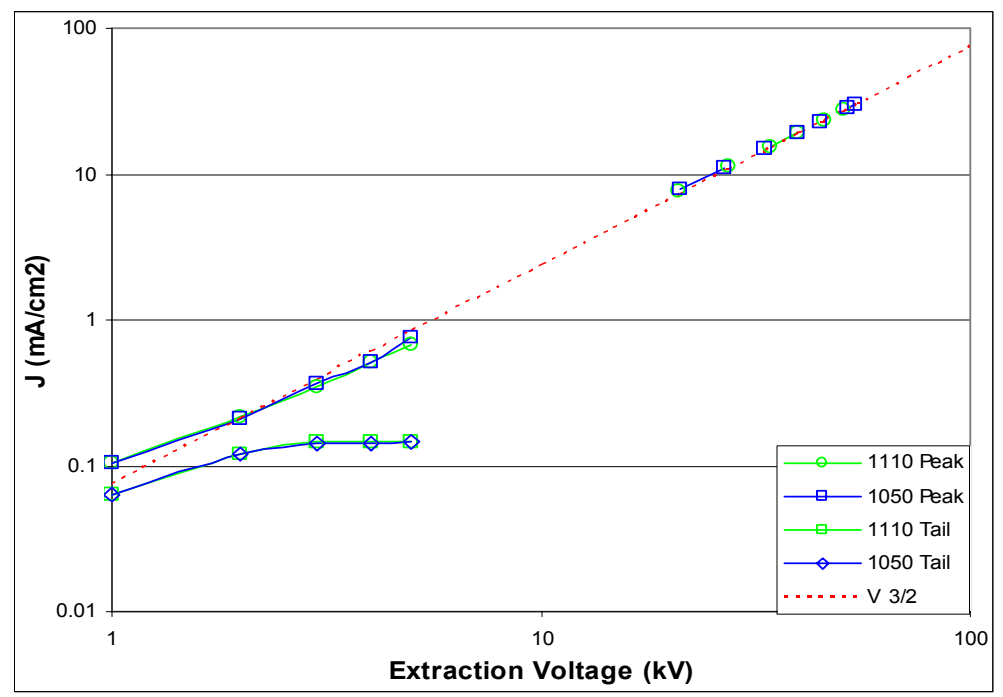

Figure 2.13. J vs $\mathrm{V}$ for Cesium source

The results for three temperatures are shown in figure 2.14. Overall the temperature required for peak current density from the Cs source is lower than that required for $\mathrm{K}$, which is expected due to the lower work function of Cs. Cs behaves in a manner much easier to predict. These results show no sign of the double decay rate seen in the $\mathrm{K}$ source. 


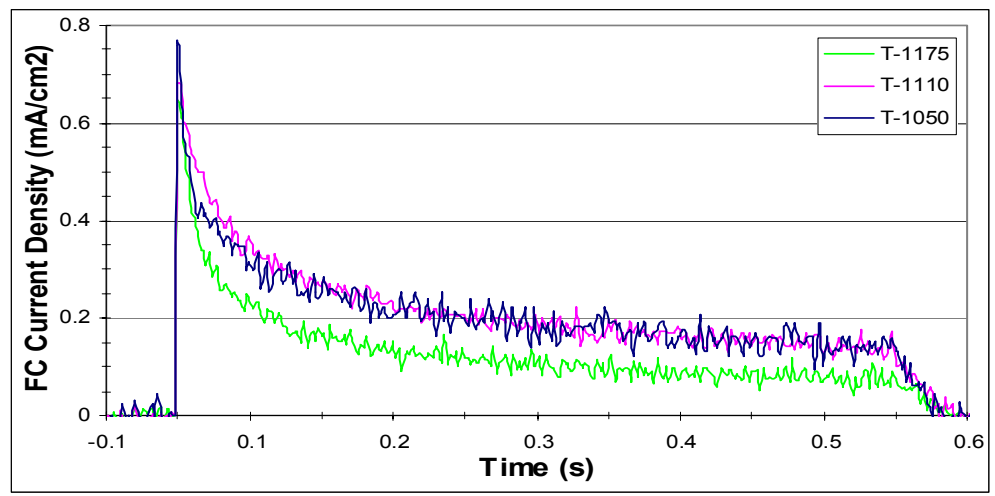

Figure 2.14. $500 \mathrm{~ms}$ Pulse at $5 \mathrm{kV}$ for 3 temperatures.

For comparison figure 2.15 shows the difference between the $\mathrm{K}$ and $\mathrm{Cs}$ sources. As expected the current for $\mathrm{Cs}$ is much lower than $\mathrm{K}$, which can easily be explained due to the equal kinetic energy but different ion mass. Each beam is given kinetic energy of:

$$
K E=\frac{1}{2} m v^{2}=5 k V
$$

However, given the different mass the velocity of each is different:

$$
\frac{v K}{v C s}=\sqrt{\frac{m C s}{m K}}=1.84
$$

Because current is charge per time, the velocity ratio is directly proportional to the magnitude of current. By comparing the initial peak values of the signals shown in figure 2.15 , this appears to hold roughly true.

$$
\frac{I_{K}}{I_{C s}}=\frac{1.4}{0.75}=1.87
$$




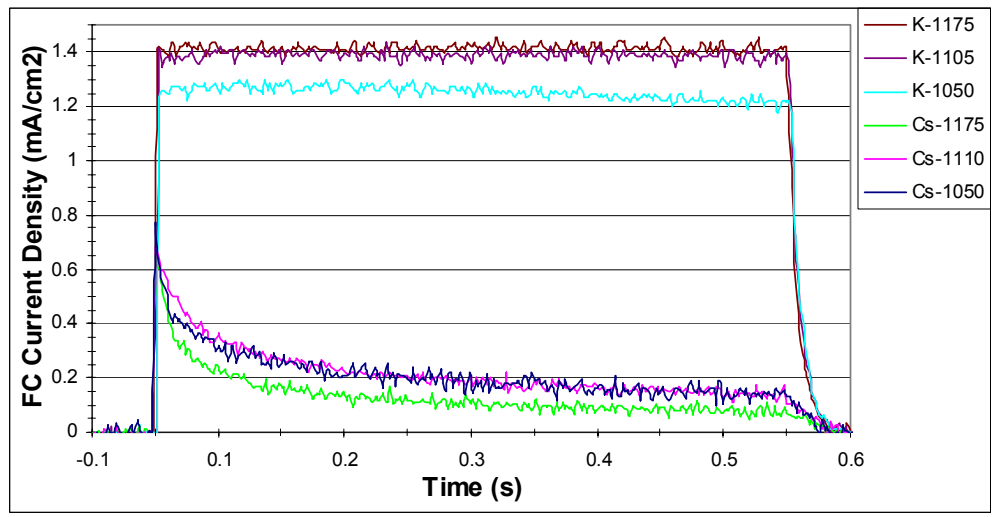

Figure 2.15. K compared to Cs

\subsection{Conclusion from Experiment}

The K alumino-silicate source is capable of long duration pulses with fairly constant high current when operated in the $0.5 \mathrm{sec}$ range. At longer pulse lengths the beam undergoes a rapid decay to a semi-steady-state condition at a current roughly $75 \%$ lower than initial value. While this is interesting for lifetime determination, it seems too low to be of any practical value. If the half second pulse is used repetitively, an important determination would be the duty cycle. At this time that study has not been conducted.

A verifiable explanation for the double decay rate of the $\mathrm{K}$ source has not yet been developed. The suspicion of contamination of the source coating can not be ruled out; in fact the result is not always repeatable with all $\mathrm{K}$ sources. Included in this report is a time-of-flight experiment used to test for such contamination. The K sources used for those tests displayed no signs of contaminates, however they also did not display the double decay rate. 


\section{Time of Flight Measurements of DC Beam}

\subsection{Motivation and Basic Principle of Experiment}

The following TOF experiments were conducted with two different setups, one with a Faraday cup, and the other with a flat plate collector. Four different alumino-silicate sources $(3-\mathrm{Cs}$, and $1-\mathrm{K})$ were used. The goal of these experiments was to determine the makeup of the beam, to insure no impurities were being emitted along with the chosen ion (K or $\mathrm{Cs})$. From previous experiments with a $\mathrm{K}$ source it was thought that perhaps impurities were causing the unusual DC beam results. During these TOF experiments, however, it became necessary to make several iterations to the setup to properly understand the results. The outcome is a better understanding of beam physics during a TOF test, and a useable design for testing the purity of an ion beam.

As a DC beam passes through a ring, a quick (100ns) pulse is applied; the time it takes for the perturbation of the beam to appear in the collector is measured. With the time, distance and ion mass data, calculations can be performed to determine the makeup of the beam.

With this understanding of the setup and results, more accurate beam energy measurements can be made by reversing the calculation and assuming the mass of the ion.

\subsection{Experimental Setups}

Each setup is shown below with brief explanations. The initial setup consisted of a timing ring and a Faraday cup with the layout shown in figure 3.1. With this setup it was very difficult to decipher the data because it was obtained with the scope in the $100 \%$ band width mode, and the capacitive noise from the ring made the signal nearly useless. 
To clean up the signal the cup opening was covered with a screen, all cables inside the vacuum chamber were heavily shielded, and the scope was switched to $20 \%$ band width. The resulting signal was significantly improved and is shown in the following results section.

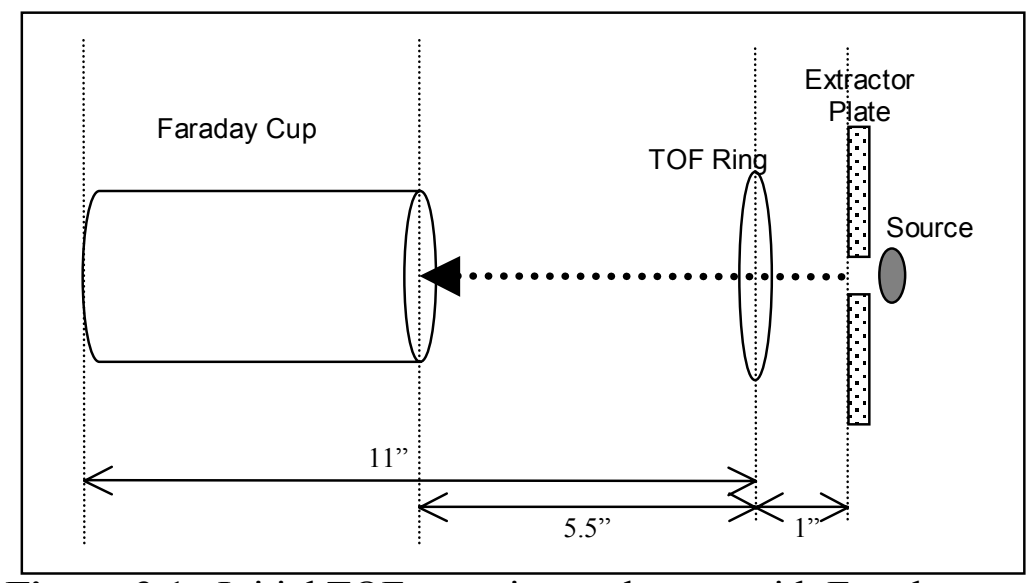

Figure 3.1. Initial TOF experimental setup with Faraday cup.

After observing results from the faraday cup setup, it was determined that a more definitive impact position was needed to obtain useful results. To resolve ambiguity, a simple flat plate was installed in place of the faraday cup as shown in figure 3.2. The plate was hooked into the scope with a $50 \Omega$ resistance.

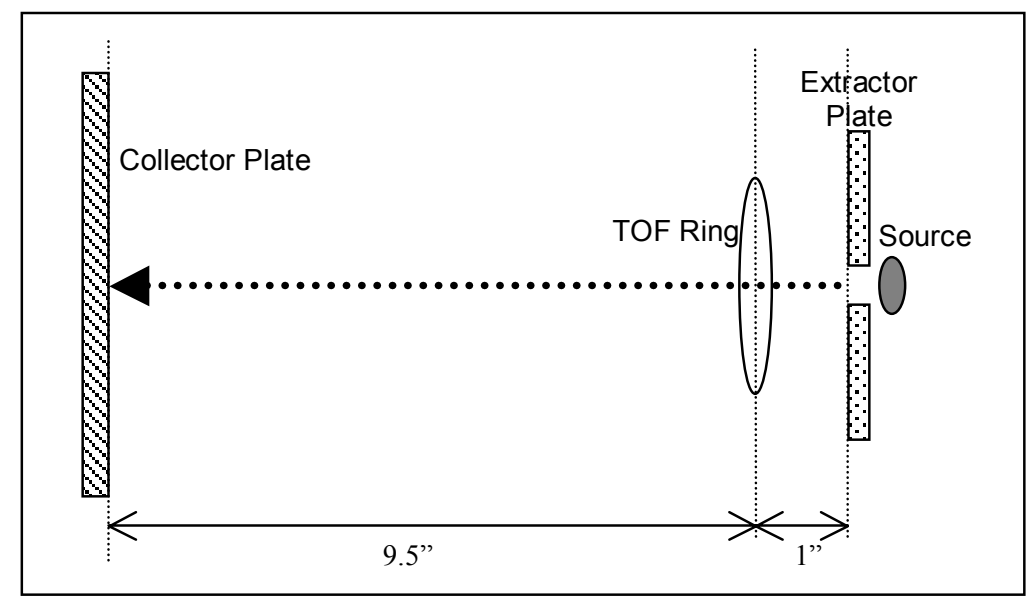

Figure 3.2. TOF experimental setup with flat plate collector.

During the development of the model shown in section 3.4, the effect of the field after the ring presented some confusion. To obtain better defined electric fields, a ground plate 
was installed after the ring (figure 3.3). Now the field after the ring is terminated in a clear plane.

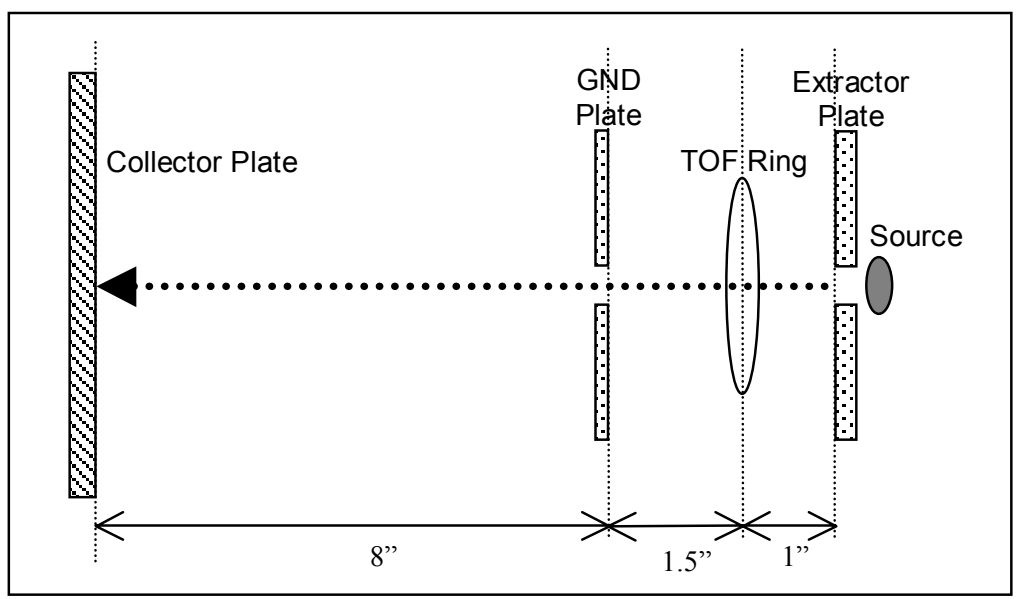

Figure 3.3. TOF setup with GND plate installed.

\subsection{Experimental Results}

With the screen installed over opening to FC the signal strength was lower but the output was cleaned up enough to see the desired timing signal. Figure 3.4 shows a composite of data taken for four different extraction voltages. The timing signal elongated and moved later in time as expected with the lower beam energies.

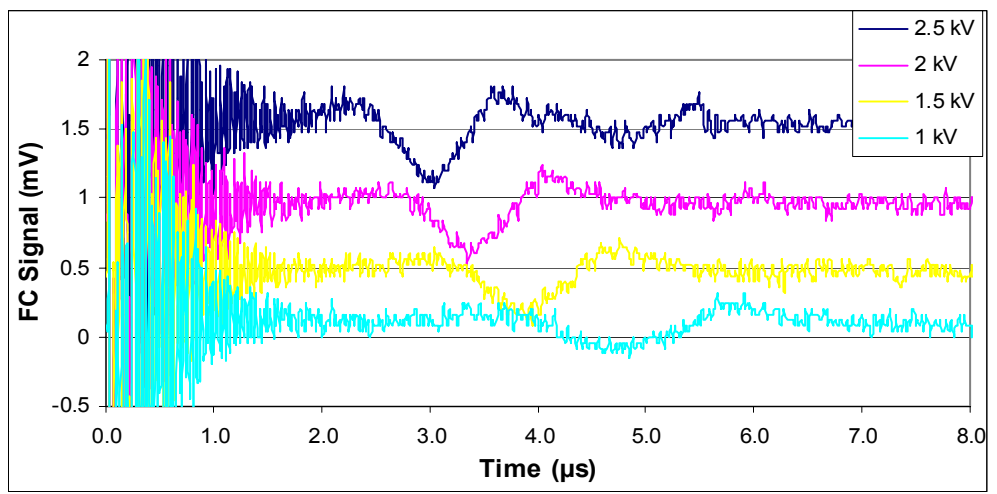

Figure 3.4. Faraday cup signals for varied extraction voltages using Cs source and $+800 \mathrm{~V}, 200 \mathrm{~ns}$ pulse on the timing ring. 
To further validate the output, the polarity of the ring voltage was reversed and as expected, the signal inverted (figure 3.5). More noticeable in this set of graphs is the second pulse further in time. This is believed to be caused by the FC geometry.

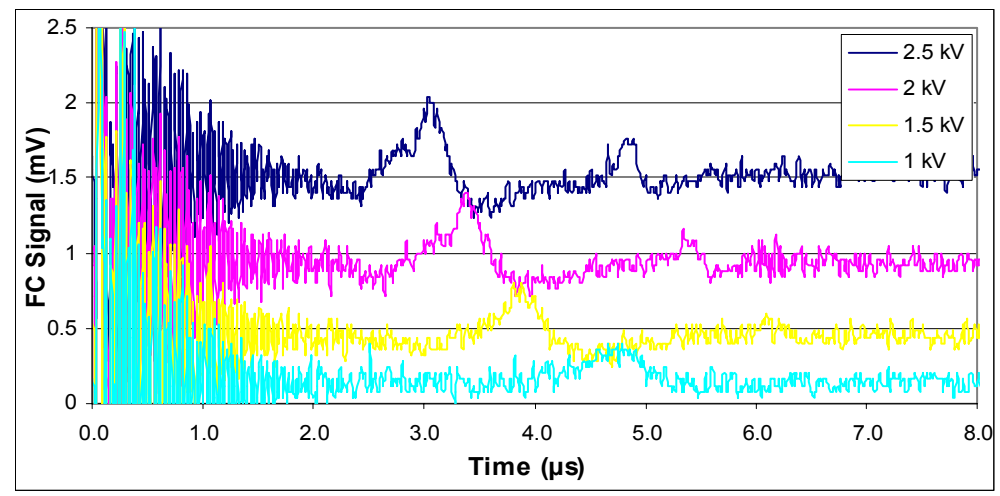

Figure 3.5. Faraday cup signals for varied extraction voltages using Cs source and $800 \mathrm{~V}, 200 \mathrm{~ns}$ pulse on the timing ring.

To illustrate this more clearly, shown in figure 3.6, are the calculated times to front edge (5.5") and the back (11") of the cup. Apparently the beam spread is significant enough at 6.5 " from the extraction plate to scrape the front edge of the cup and cause the first timing pulse. Then subsequently the remainder of the beam impacts the back of the cup causing the later pulse.

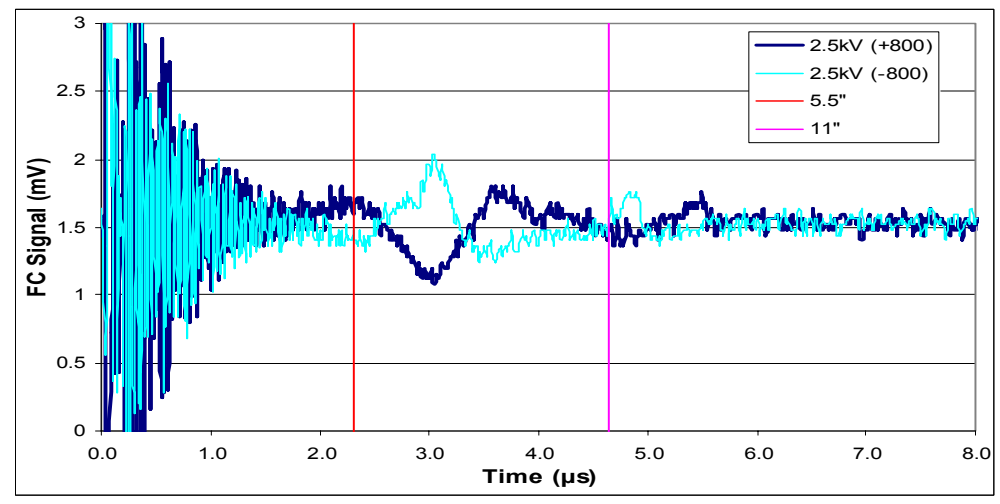

Figure 3.6. TOF pulses for Cs. Positions for 5.5" and 11 " were calculated and labeled to show correlation with timing signals.

For the calculations the following simple energy equation and mass data was used: 


$$
q E=\frac{m v^{2}}{2} ; v=\frac{d}{t}
$$

\begin{tabular}{|c|c|c|}
\hline Ion & Atomic mass & mass kg \\
\hline Cesium Cs & 132.90545 & $2.207 \times 10^{-25} \mathrm{~kg}$ \\
\hline Potassium K & 39.0983 & $6.493 \times 10^{-26} \mathrm{~kg}$ \\
\hline
\end{tabular}

As can be seen from figure 3.6, the measurement point is at the beginning of the perturbation. This point of the signal is used for all subsequent measurements. By conducting this measurement for all beam energies and plotting versus calculations, figure 3.7 shows the close correlation between the two. Error for these measurements is all below 3\%

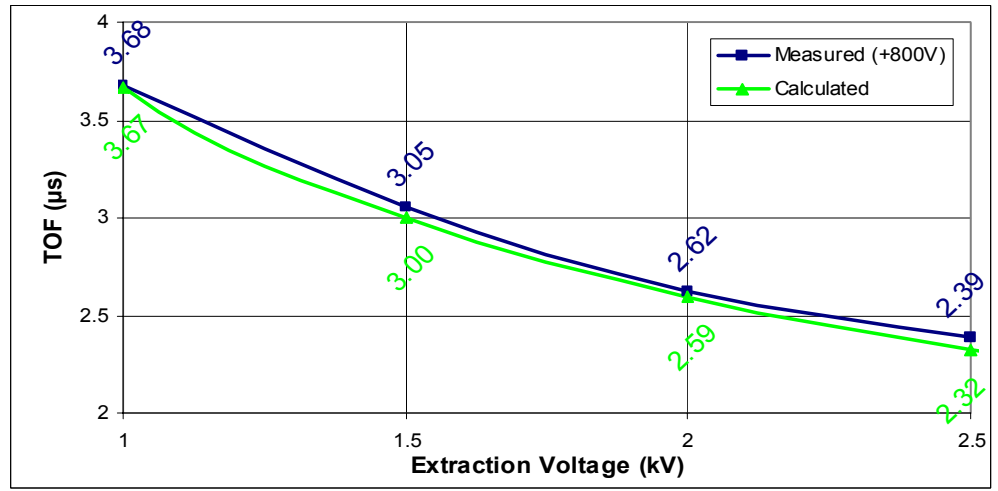

Figure 3.7. Cs source - Comparison of pulse timing to calculation for the front of FC (5.5 inches).

The following figures $(3.8-3.10)$ show the results from a K source using the Faraday cup. These differ, as expected, from the Cs source in both amplitude and timing. The lighter $\mathrm{K}$ ion travels at a higher velocity resulting in the higher current. The predicted values for timing are shown compared to the measured values in figure 3.10. 


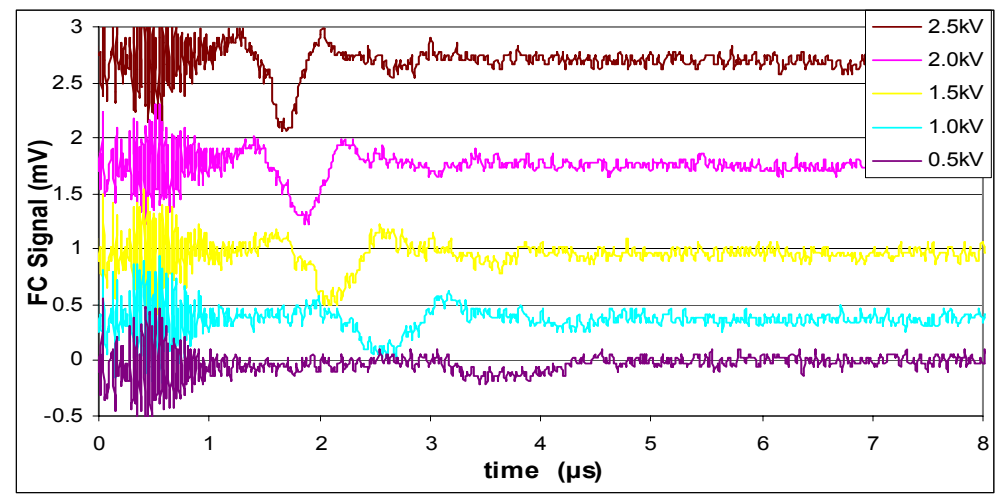

Figure 3.8. Faraday cup signals for varied extraction voltages using K source and $+800 \mathrm{~V}, 200 \mathrm{~ns}$ pulse on the timing ring.

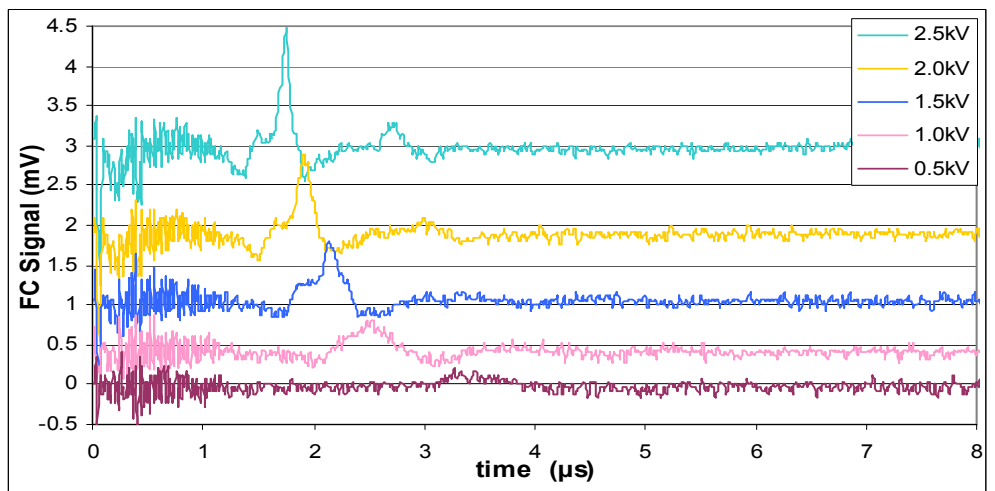

Figure 3.9. Faraday cup signals for varied extraction voltages using K source and $-800 \mathrm{~V}, 200 \mathrm{~ns}$ pulse on the timing ring.

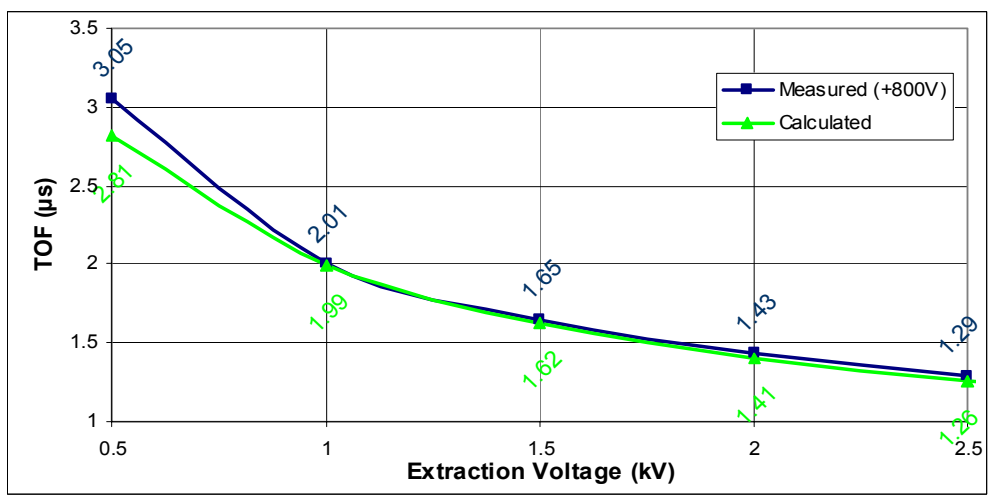

Figure 3.10. Measured signal plotted versus calculated for K using the Faraday cup.

All measurements in figure 3.10 except the one taken at $0.5 \mathrm{kV}$ were below $3 \%$ error. The $0.5 \mathrm{kV}$ measurement was off by almost $8 \%$; however this is primarily due to measurement uncertainty. 
Once again to illustrate the problem with the Faraday cup, figure 3.11 shows the appearance of multiple pulses. The different pulses were correlated to different locations on the faraday cup for both Cs and K. The first large pulse correlated, by calculation, with the opening of the FC, while the second pulse is thought to be the back of the cup.

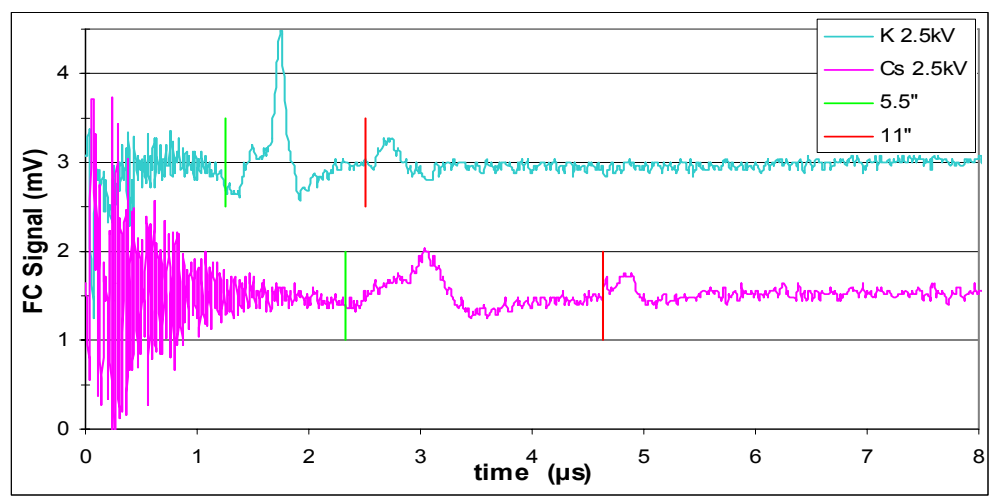

Figure 3.11. $\mathrm{K}$ and $\mathrm{Cs}$ signals shown together to illustrate difference in current magnitude and evolution of the timing wave. For both the Faraday cup measurements correlate to pulses.

The next set of figures show the results using a K source and the flat-plate collector. As expected, only one pulse appeared with this setup, and properly correlated, to the measured distance.

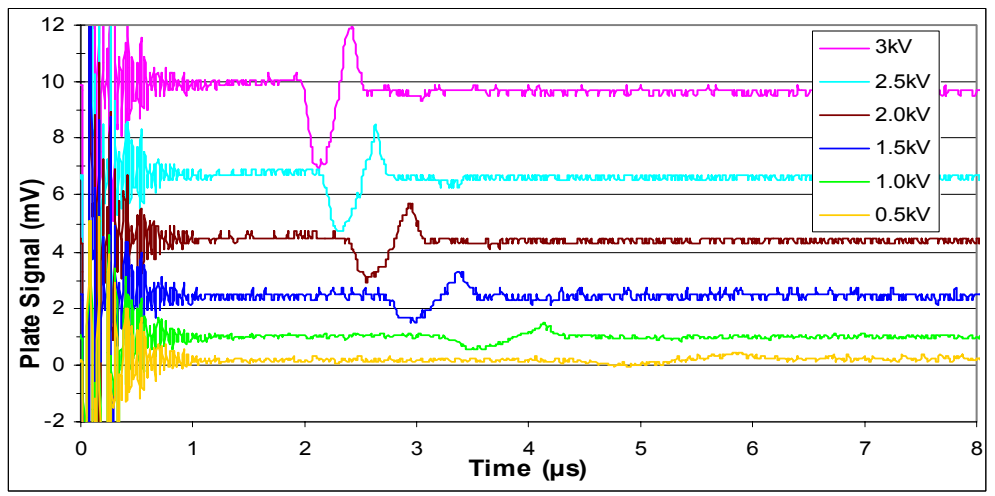

Figure 3.12. Flat plate collector signals for varied extraction voltages using $\mathrm{K}$ source and $+800 \mathrm{~V}, 100 \mathrm{~ns}$ pulse on the timing ring. 


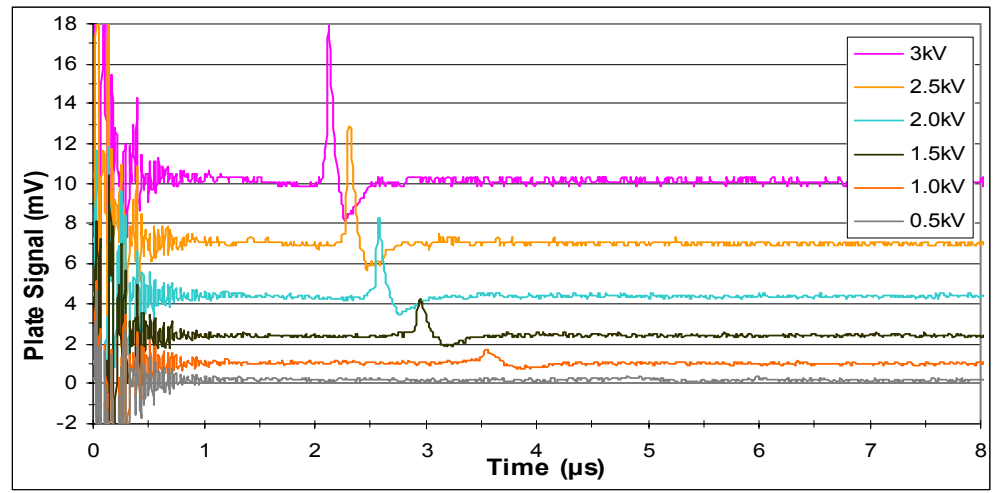

Figure 3.13. Flat plate collector signals for varied extraction voltages using $\mathrm{K}$ source and $-800 \mathrm{~V}, 100 \mathrm{~ns}$ pulse on the timing ring.

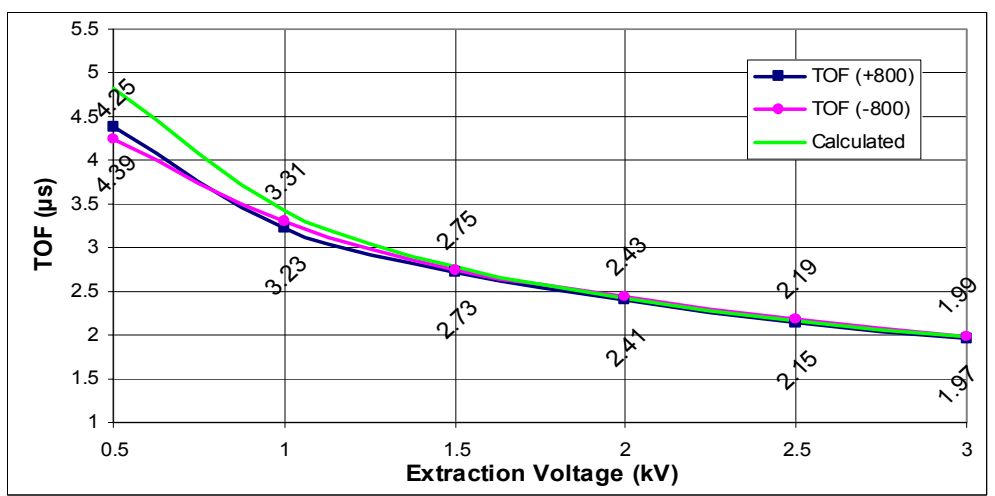

Figure 3.14. Measured signal plotted versus calculated for $\mathrm{K}$ using the flat-plate collector.

Except for the shape of the wave, the results using the GND plate, in terms of timing, were very similar to those obtained with out the plate. The difference in the resulting signal is shown in figure 3.15. The ground plate condenses the field after the ring causing a more pronounced acceleration of the particles located between the ring and plate during the timing pulse. Consequently, with this setup, a leading hump is produced. 


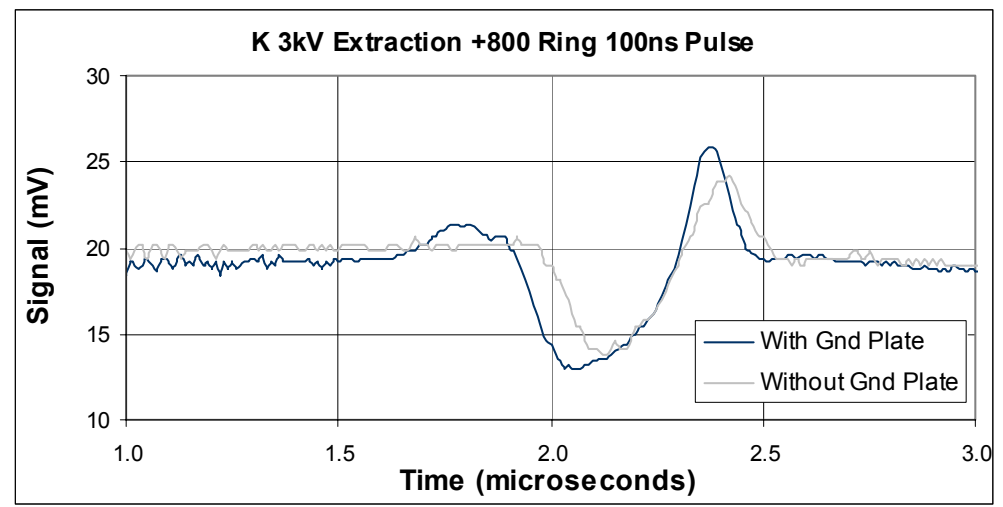

Figure 3.15. Difference in signal from installing the ground plate. This case shows the positive $800 \mathrm{~V}$ ring pulse.

In fact the distortion of the pulse characteristics becomes more prevalent as the timing pulse is increased. This is as expected due to more significant affect the longer or higher voltage has on the beam as it passes through the resulting field. A sample of the effects is seen in figure 3.16. A more detailed explanation is covered in the model section.

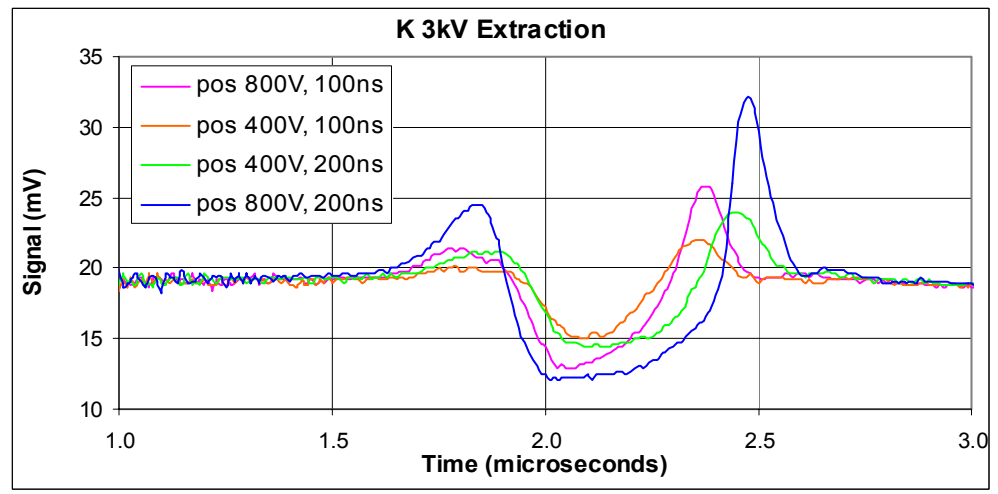

Figure 3.16. Four different conditions shown together to illustrate the effect of a change in each parameter.

\subsection{Development of Model and Comparison to Measured Data}

In order to obtain an analytic model to describe the resulting signal, I treated a finite ensemble of particles with simple classical mechanics. By calculating the effects of the fields on a particle by particle basis I was able to predict the time of impact with the 
collector plate for each individual particle given its initial position. Then by assigning a "current" magnitude by summing the number of particles that arrived at a given time, I was able to nearly reproduce the signal seen on the collector plate. With this model several simplification are made. The electric fields are treated as if between uniform parallel plates, space charge effects are ignored, and no account is made for beam divergence.

With space charge limited flow the expression for $E$ should be $E=-V_{o}(z / d)^{4 / 3}$. For these DC beam tests, current and space-charge effects are assumed to be sufficiently low to use assumption that $\mathrm{E}=-\mathrm{V}_{\mathrm{o}} / \mathrm{d}$. However, even with these gross simplifications, the model predicts with a fair amount of accuracy the resultant signal. The overall goal of this exercise was to gain an understanding of how the particles are moving to allow for more accurate TOF measurements. Figures 3.17 - 3.19 show the comparison of calculated vs. real for three different conditions.

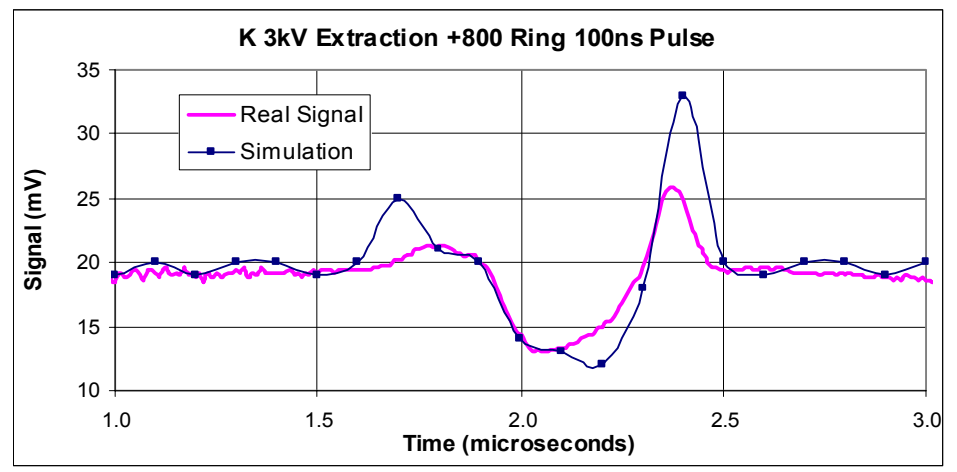

Figure 3.17. Comparison of real signal to calculation results using a 100ns positive $800 \mathrm{~V}$ pulse on the timing ring with the ground plate installed. 


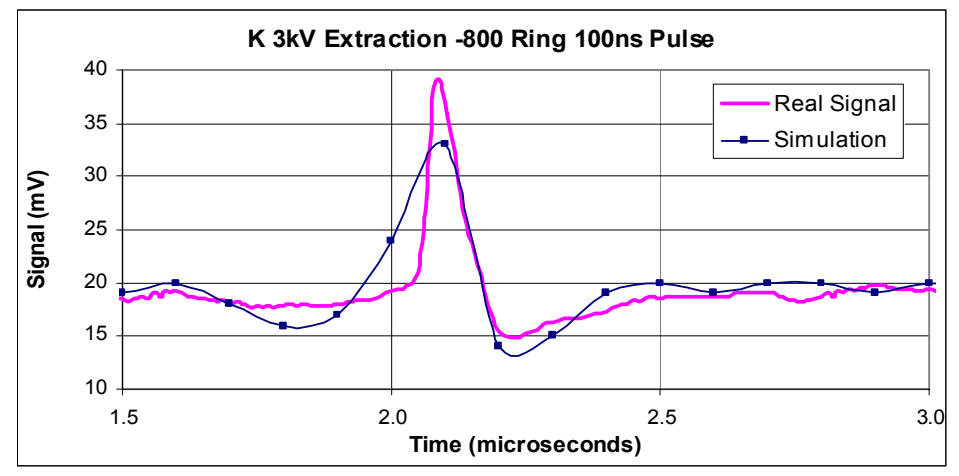

Figure 3.18. Comparison of real signal to calculation results using a 100ns negative $800 \mathrm{~V}$ pulse on the timing ring with the ground plate installed.

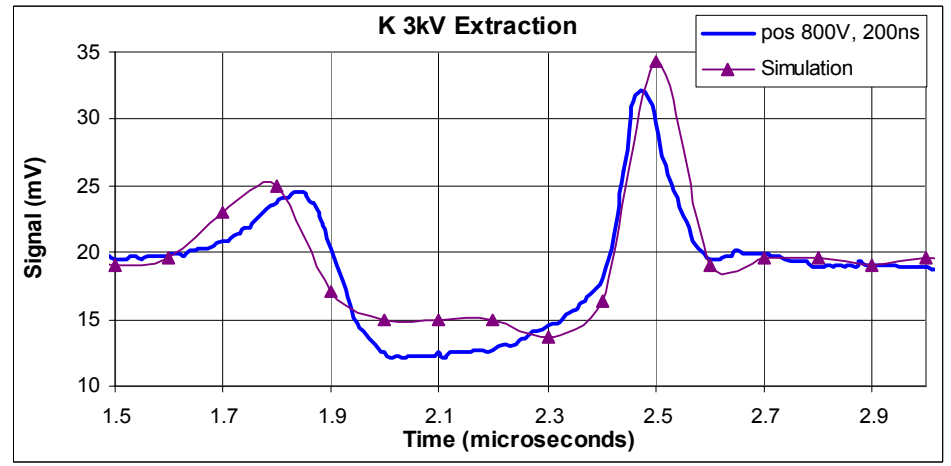

Figure 3.19. Comparison of real signal to calculation results using a 200ns positive $800 \mathrm{~V}$ pulse on the timing ring.

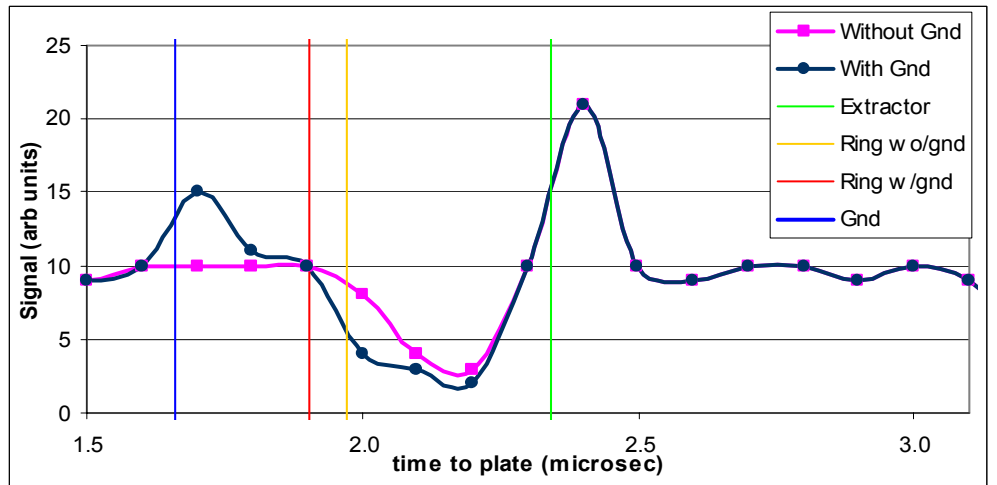

Figure 3.20. Simulation showing resulting positions in relation to initial position of particle for with and without the ground plate.

The important features of the waveform can be easily explained by using the simple model in figure 3.21. The bunched particles create the peaks and gaps produce the trough. Without the ground plate the leading bunch is not present. 


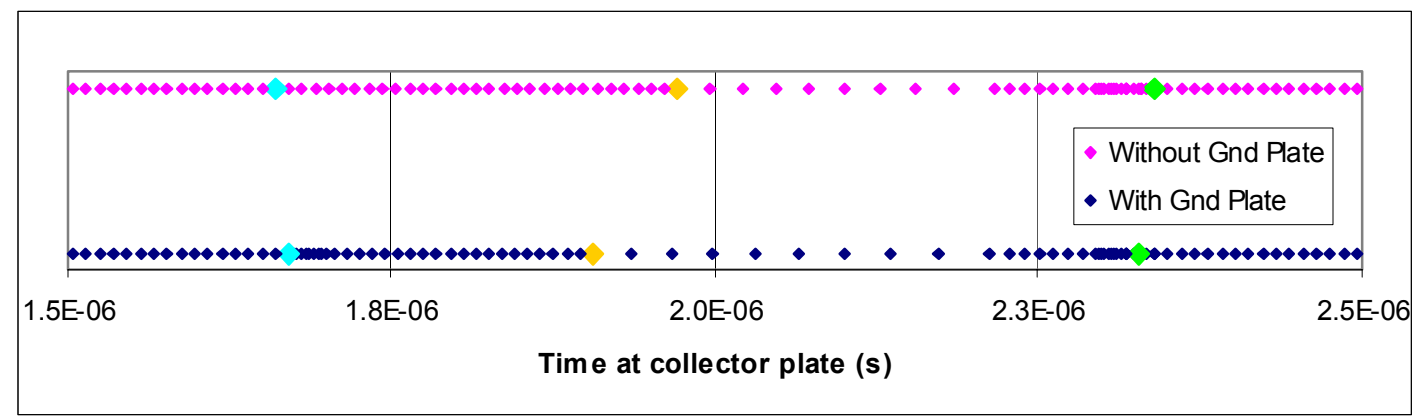

Figure 3.21. Simulated view of beam showing effect on charge density.

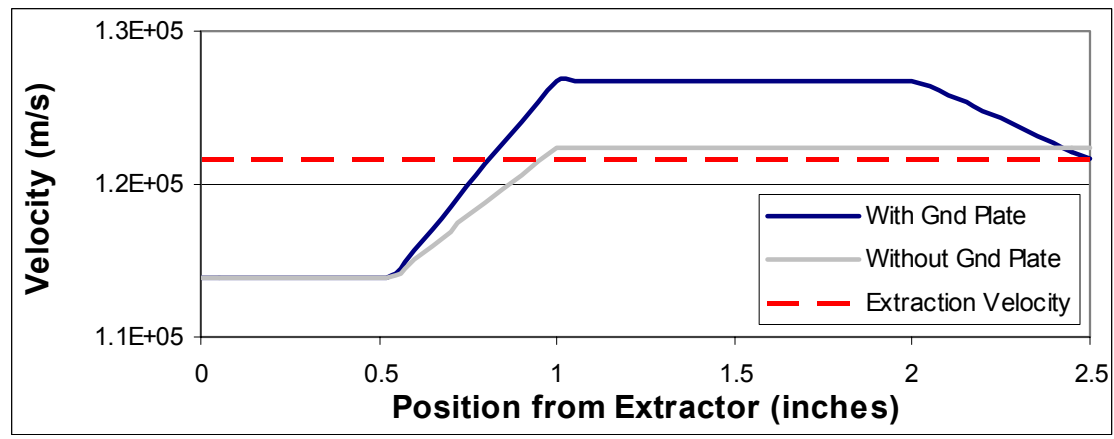

Figure 3.22. Particle velocity at collector plate shown versus initial position at the beginning of the timing pulse.

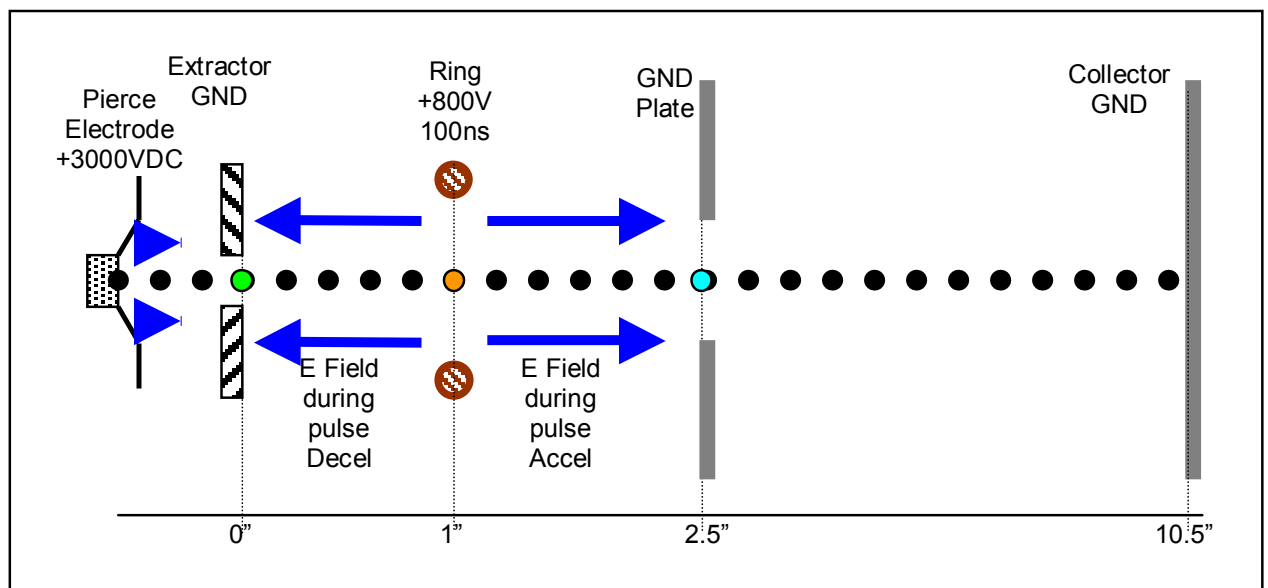

Figure 3.23. TOF setup showing geometry used for calculations.

The following equations are used in the development of the model.

$$
F=q\left(\vec{E}+\frac{v}{c} \times \vec{B}\right)=m a
$$

By assuming magnetic fields are negligible the Lorentz force reduces simply, allowing the following to be used: 


$$
\begin{gathered}
q E=\frac{m v^{2}}{2} \\
v_{o}=\sqrt{\frac{2 q V}{m}}
\end{gathered}
$$

The electric fields generated during the pulse have been significantly simplified by assuming infinite plane geometry with no effect from space-charge.

$$
\begin{aligned}
& \vec{E}=-\vec{\nabla} \Phi \\
& E_{x}=-\frac{V_{o}}{s}
\end{aligned}
$$

With this simple field model the following expression is used for particle acceleration in the gaps.

$$
a=\frac{q V}{d m}
$$

This is then used in the basic equations of motion.

$$
\begin{gathered}
s=s_{o}+\frac{a t^{2}}{2}+v_{o} t \\
v=v_{o}+a t
\end{gathered}
$$

\subsection{Contaminated Cs source}

During the testing of the sources, two Cs sources were found to clearly have contamination of some sort. Figure 3.24 shows a confusing signal that resulted from the first problem source. This measurement was taken using a Faraday cup. 


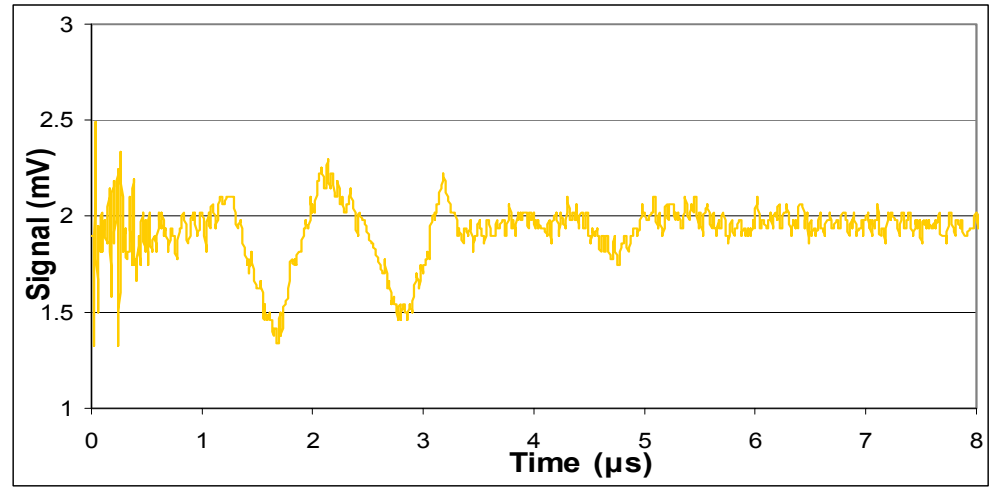

Figure 3.24. Contaminated Cs signal measured with FC setup.

The first two large pulses can easily be matched with data taken from clean sources to help identify the peaks. By overlaying the signals as shown in figure 3.25 , the earlier peak can be correlated with a $\mathrm{K}$ timing signal. This indicates that in addition to Cs ions, $\mathrm{K}$ ions are also being emitted from this source.

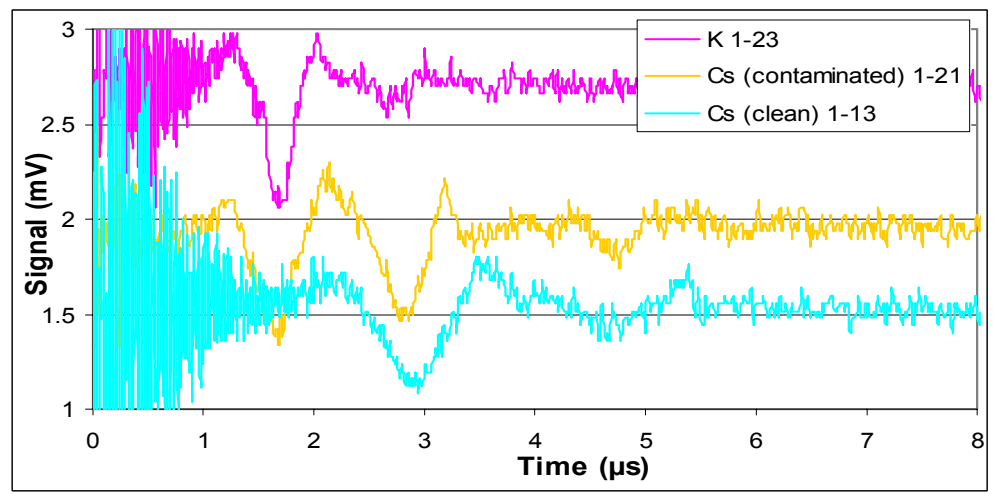

Figure 3.25. Contaminated Cs signal compared to the clean Cs source and a K source. All measured with FC setup.

For the next series of measurements a Cs source was used with the gnd plate TOF setup. From the signal seen in figure 3.26 it is apparent that the beam contains ions of two separate masses. The second and larger pulse occurring at $\sim 3.5 \mu$ s is clearly Cs, while it appears that the contaminant is $\mathrm{K}$. 


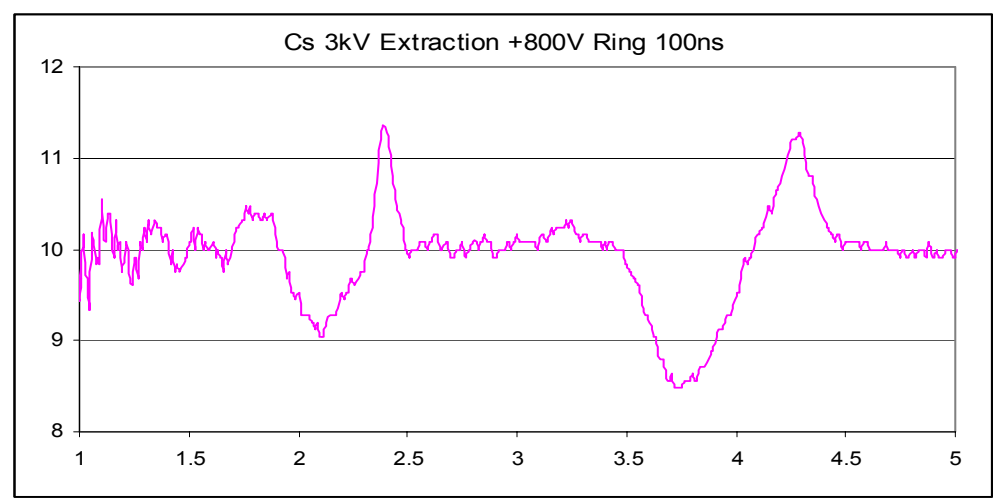

Figure 3.26. Cs source signal showing two timing pulses.

Figure 3.27 shows a comparison of $\mathrm{K}$ beam data to the contaminated Cs data. As can be seen from the figure the contaminant is very likely $\mathrm{K}$.

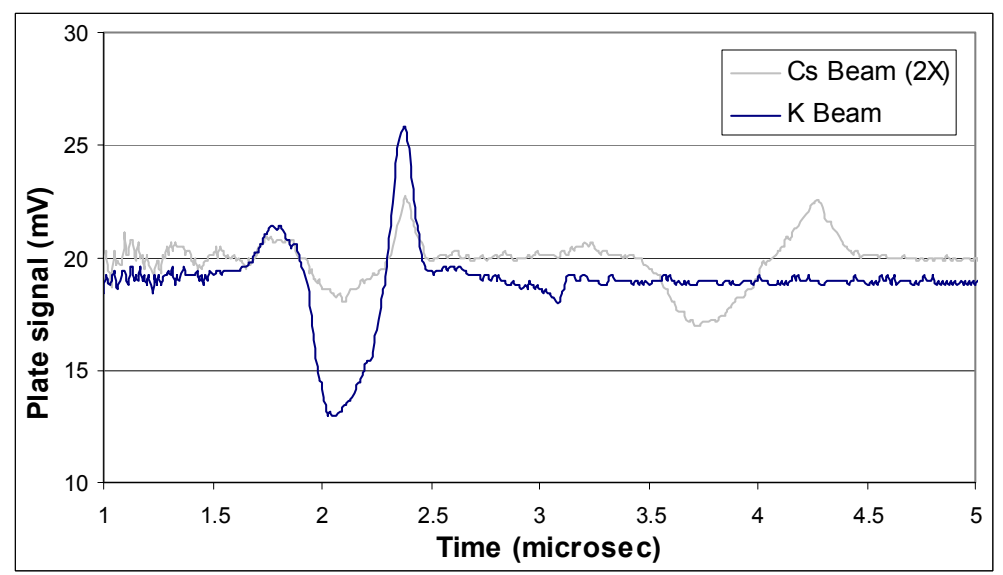

Figure 3.27. K beam data superimposed on the contaminated Cs beam data. Cs amplitude is doubled for easier comparison.

For situations like this where the mass difference between ions is significant, it is easy to distinguish multiple ions in the beam. If more sensitivity is needed then the distance to the collector plate can be increased for greater separation. With this setup it can be expected that a difference in timing greater or equal to $0.5 \mu$ sould allow discrimination between ions. $0.5 \mu$ s difference can be put into perspective with the following rough calculation:

$$
v=\sqrt{\frac{2 q V}{m}}=\frac{d}{t}
$$




$$
\Delta t=t_{1}-t_{2}=\frac{d}{\sqrt{2 q V}}\left(\sqrt{m_{1}}-\sqrt{m_{2}}\right)
$$

As long as $\Delta t \geq 0.5 \mu$ s the pulse separation should be sufficient, therefore:

$$
(\sqrt{m 1}-\sqrt{m 2}) \geq 5.8 \times 10^{-14} \mathrm{~kg}
$$

If considering alkali metals and the mass separation between each, it would be possible with this TOF setup to decipher between each if present in the beam.

\subsection{Conclusions from Experiment}

These experiments and the developed model provide an easy and accurate method for determining the make-up of an ion beam. Shown in several instances, changing aluminosilicate sources introduces the possibility of cross contamination. The presence of undesired ions in a beam can adversely affect the quality and optics of the resulting beam. This apparatus provides practical test to determine the source purity. Another valuable application for this setup is the measurement of beam energy. The important detail derived from this series of experiments is an understanding of where and how to measure the timing pulse. When using a positive timing pulse, the moment the signal begins to sharply drop is when the ions that were in the ring are hitting the collector. Some confusion has been observed regarding this measurement; however the above results clearly demonstrate this theory. 


\section{Alumino-Silicate Source Lifetime Experiments}

\subsection{Setup and Method of Measurement}

For this series of experiments the DC pulser was used. In place of the signal generator a DC voltage was supplied to the IGBT drivers to trigger and maintain a constant extraction voltage for the duration of each shot. A Faraday cup was used to measure beam current. For all tests a $5 \mathrm{kV}$ extraction was used with $500 \mathrm{~V}$ on the cup suppressor ring. The oscilloscope in each case was set to record at $20 \mathrm{~s} / \mathrm{div}$, and for the longest case the scope was capable of recording 2400div (13.33hrs).

Of particular interest with these tests is the percent of alkali atoms removed from the original concentration, the length of usable service life, and the characteristics over the lifetime.

\subsection{Experimental Results}

For the following results four separate $\mathrm{K}$ sources were completely depleted. Beam current was recorded for the duration and is shown for each test. Two of the sources were coated to a thickness of 16mil, and the other two were given half coats of only $8 \mathrm{mil}$. Each test was initiated with a fresh source and the shots were repeated until the no further beam could be extracted. Calculations are made to determine the performance of each source using this data:

- Coating is $\mathrm{K}_{2} \mathrm{OAl}_{2} \mathrm{O}_{3} \cdot 4 \mathrm{SiO}_{2}$

- $G M W$ of coating $=436.49588 \mathrm{~g} / \mathrm{mole}$

- $\quad$ mass of $\mathrm{K}=39.096 \mathrm{~g} / \mathrm{mole}=6.49 \times 10^{-26} \mathrm{~kg}$

- $N_{A}=6.022 \times 10^{23}$ particles $/$ mole

- $q=1.6022 \times 10^{-19} \mathrm{C}$

For each source the total $\mathrm{K}_{2} \mathrm{OAl}_{2} \mathrm{O}_{3} \cdot 4 \mathrm{SiO}_{2}$ molecules and $\mathrm{K}$ atoms contained in the coating before test are calculated: 


$$
N=\frac{m N_{A}}{G M W} ; N_{K}=2 N
$$

The mass of the coating is measured during fabrication of each source. Then by comparing pre-test weight to a second measurement is made after the test, the change in mass as a result of the ion emission is determined. Current versus time is recorded and shown for each test in figures $4.1-4.3$. From these plots integration is performed to determine the total $\mathrm{A} \cdot \mathrm{s}$ removed from each source. Using this value a calculation is made to determine the number of $\mathrm{K}$ ions removed and collected in the Faraday cup. By comparing the number of beam ions collected to the reduction of mass, a percentage of total $\mathrm{K}$ inventory is calculated. A summary of this data is shown in table 4.1. While optimistic, for these tests it is assumed that current due to secondary electrons is small for these extraction voltages and therefore ignored.

Test 1 conducted on the 16 mil $K$ source (4/6-4/8):

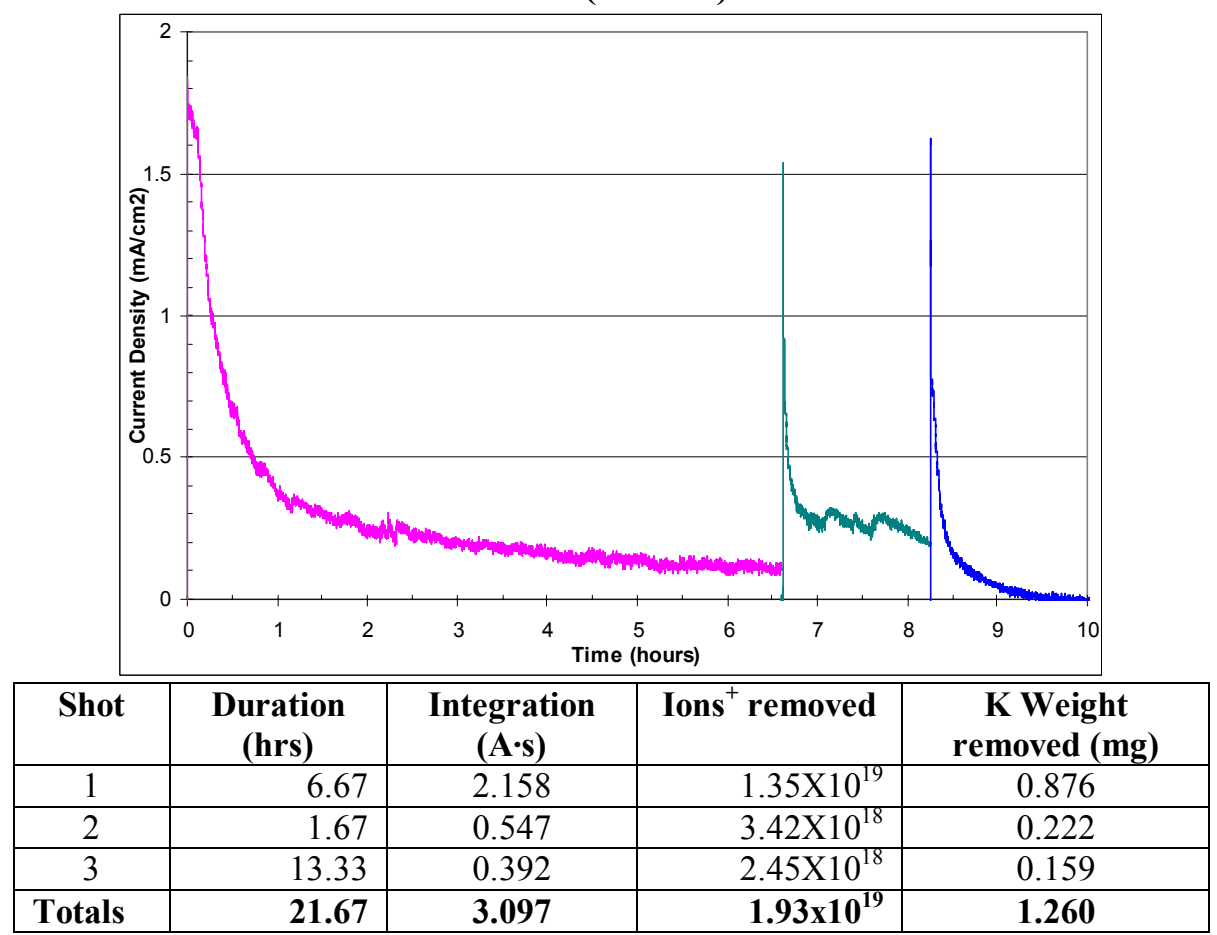

Figure 4.1. Test 1: shot 1 (6.67hrs), shot 2 (1.67hrs), shot 3 (13.33hrs only $2 \mathrm{hrs}$ shown). 
Test 2 conducted on 8 mil $K$ source (4/16):

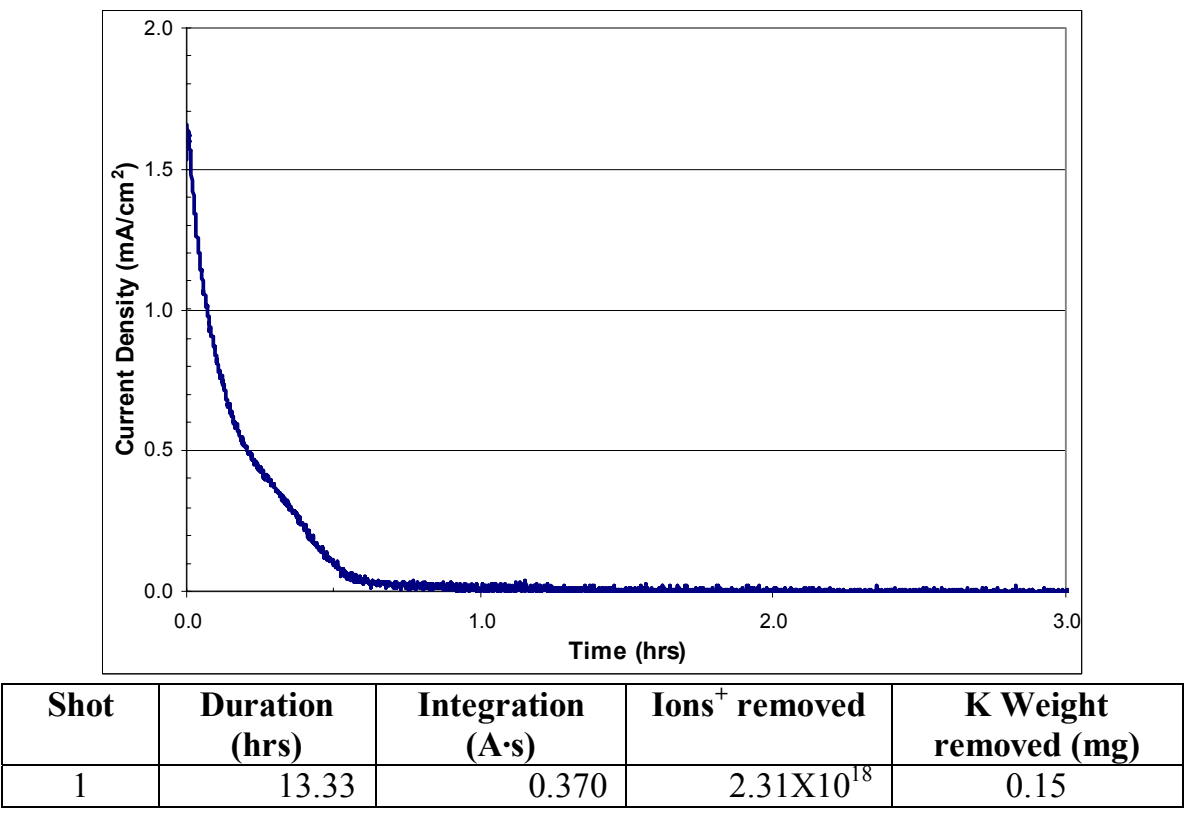

Figure 4.2. Test 2: shot 1 (13.33hrs) only first 3 hrs shown.

Test 3 conducted on the 16 mil $K$ source (4/21-4/22):

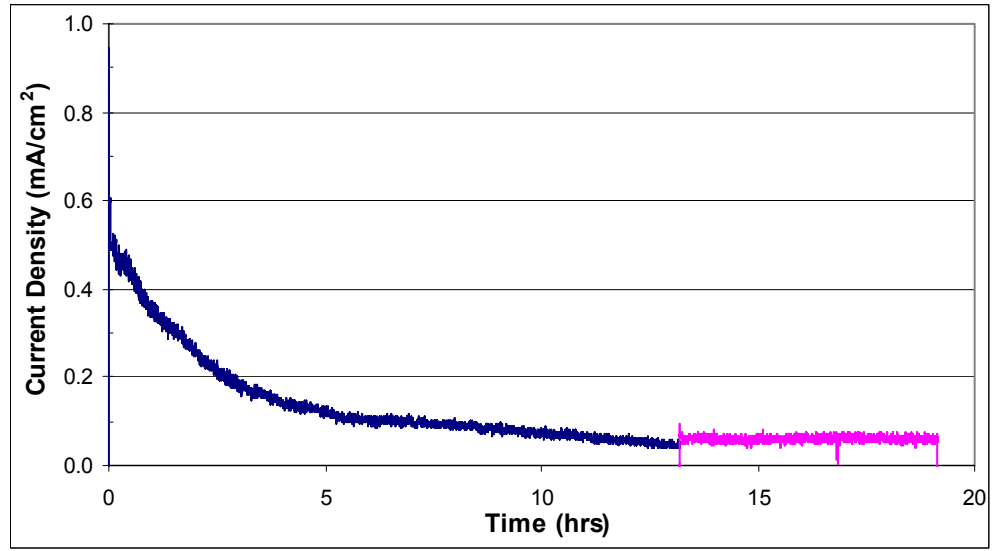

\begin{tabular}{|c|c|c|c|c|}
\hline Shot & $\begin{array}{c}\text { Duration } \\
\text { (hrs) }\end{array}$ & $\begin{array}{c}\text { Integration } \\
\mathbf{( A \cdot s )}\end{array}$ & Ions $^{+}$removed & $\begin{array}{c}\text { K Weight } \\
\text { removed (mg) }\end{array}$ \\
\hline 1 & 13.33 & 2.191 & $1.37 \times 10^{19}$ & 0.89 \\
\hline 2 & 6.00 & 0.377 & $2.36 \times 10^{18}$ & 0.15 \\
\hline Totals & $\mathbf{2 1 . 6 7}$ & $\mathbf{2 . 5 6 8}$ & $\mathbf{1 . 6 0 3 \times 1 0}^{19}$ & $\mathbf{1 . 0 4}$ \\
\hline
\end{tabular}

Figure 4.3. Test 3: shot 1 (13.33hrs) and shot 2 (6hrs) Not visible on this time scale, current density began at $0.95 \mathrm{~mA} / \mathrm{cm}^{2}$. 
Test 4 conducted on the 8 mil K source (4/26):

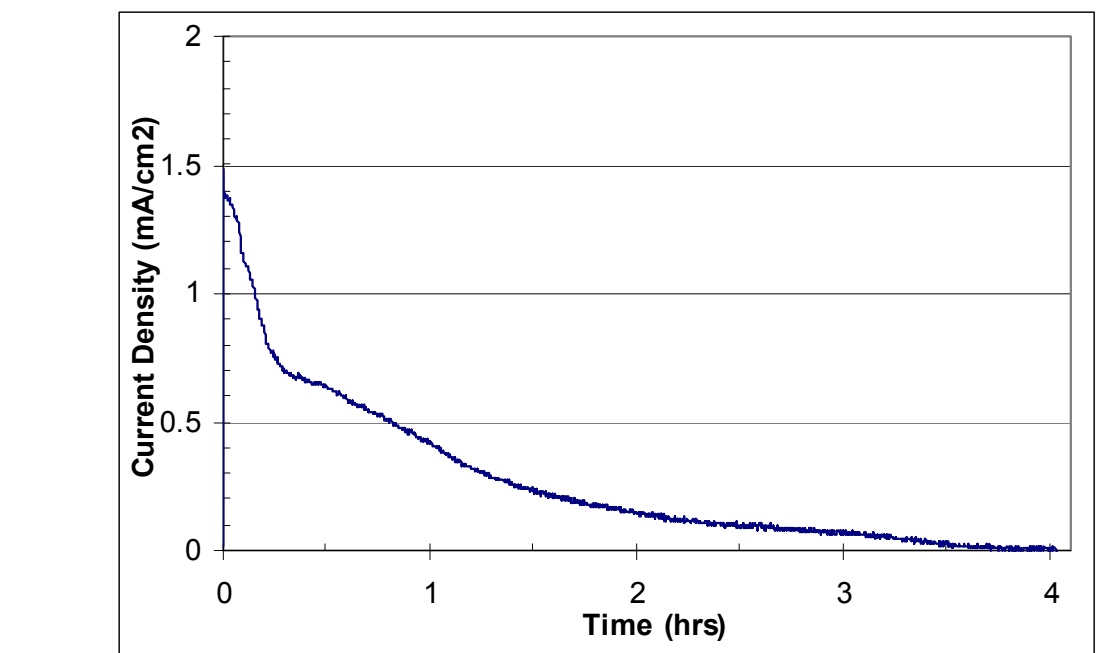

\begin{tabular}{|c|c|c|c|c|}
\hline Shot & $\begin{array}{c}\text { Duration } \\
(\mathbf{h r s})\end{array}$ & $\begin{array}{c}\text { Integration } \\
\mathbf{( A \cdot s )}\end{array}$ & Ions $^{+}$removed & $\begin{array}{c}\text { K Weight } \\
\text { removed (mg) }\end{array}$ \\
\hline 1 & 13.33 & 1.232 & $7.70 \times 10^{18}$ & 0.5 \\
\hline
\end{tabular}

Figure 4.4. Test 4: shot 1 (13.33hrs) only first four hours shown.

\begin{tabular}{|c|c|c|c|c|c|c|c|c|}
\hline Test & $\begin{array}{c}\text { Total } \\
\text { Extraction } \\
\text { Duration } \\
\text { (hrs) }\end{array}$ & $\begin{array}{c}\text { Thickness } \\
\text { of coating } \\
\text { (mil) }\end{array}$ & $\begin{array}{c}\text { Mass of } \\
\text { coating } \\
\text { (mg) }\end{array}$ & $\begin{array}{c}\text { Initial } \\
\text { mass of } \\
\text { K (mg) }\end{array}$ & $\begin{array}{c}\text { Change } \\
\text { in mass } \\
\text { from test } \\
\text { (mg) }\end{array}$ & $\begin{array}{c}\text { Mass } \\
\text { removed } \\
\text { as K ions } \\
\text { (mg) }\end{array}$ & $\begin{array}{c}\text { K ions } \\
\text { removed } \\
\text { from initial } \\
\text { K inventory }\end{array}$ & $\begin{array}{c}\text { Mass } \\
\text { lost as } \\
\text { K ions }\end{array}$ \\
\hline $\mathbf{1}$ & 21.67 & 16 & 19.50 & 3.50 & 2.00 & 1.26 & $36 \%$ & $63 \%$ \\
\hline $\mathbf{2}$ & 13.33 & 8 & 8.00 & 1.43 & 1.30 & 0.15 & $11 \%$ & $12 \%$ \\
\hline $\mathbf{3}$ & 19.33 & 16 & 17.40 & 3.12 & 0.90 & 1.04 & $33 \%$ & $116 \%$ \\
\hline $\mathbf{4}$ & 13.33 & 8 & $?$ & $?$ & $?$ & 0.50 & $?$ & $?$ \\
\hline
\end{tabular}

Table 4.1. Summary of results from four tests.

While conducting these tests, concern developed regarding the possibility that the Faraday cup is missing a portion of the beam. By conducting a simple current analysis of the system during this test, it appeared that the current flow on the negative power supply indicated the beam was striking the extractor plate. From figure 4.5 it seems apparent that any current flow monitored on the negative power supply is clear evidence of beam loss.

$$
\begin{gathered}
i_{\text {beam }}=i_{\text {pos }}-i_{\text {neg }} \\
i_{\text {beam }}=i_{\text {signal }}=\frac{V_{\text {signal }}}{50 \Omega}
\end{gathered}
$$




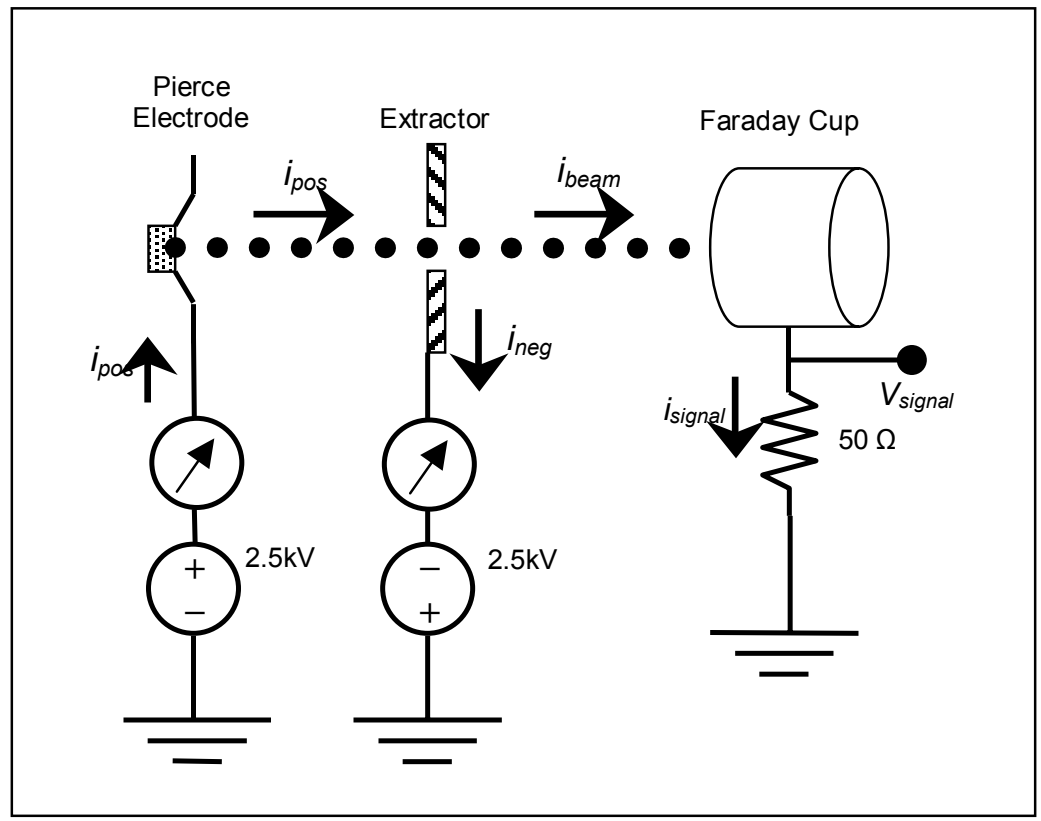

Figure 4.5. Source diagram labeled with current flow.

After further investigation it was determined that the current from the power-supplies was a function of the system and not a result of the beam. To demonstrate this, the data for figure 4.6 was taken with and without beam.

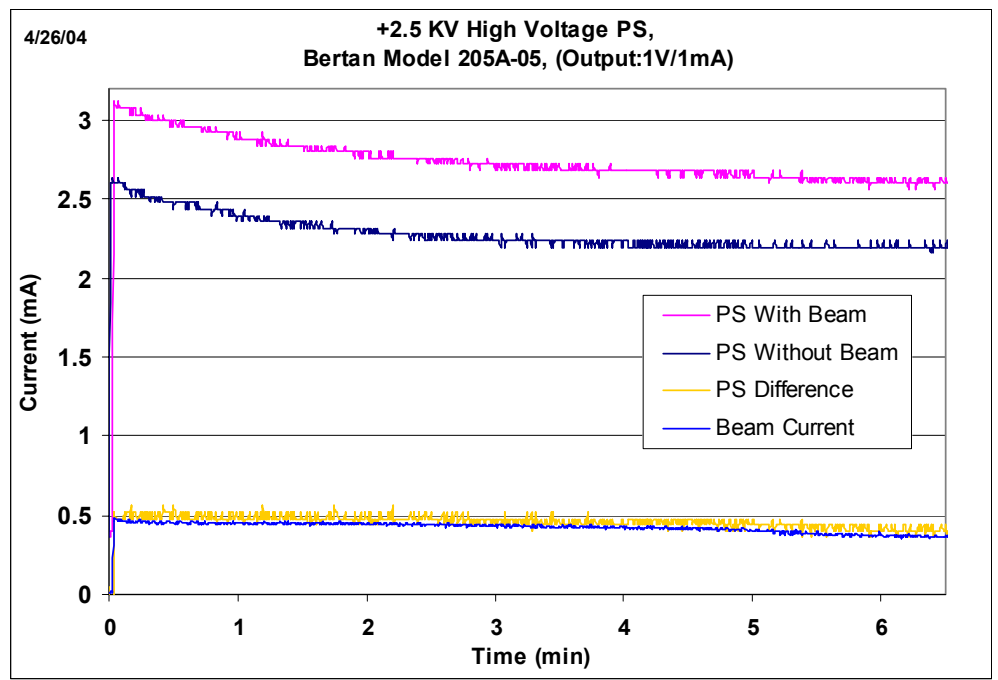

Figure 4.6. Current from power supply compared to beam current.

This also shows that the assumption made regarding secondary electron current is actually reasonably valid. 


\subsection{Conclusions}

At this time a definitive conclusion can not be drawn from these experiments.

Further investigation is required to more thoroughly understand the results. Preliminarily it appears that a low percentage $(\sim 30 \%)$ of the initial $\mathrm{K}$ inventory is available for

extraction. However, if given that this percentage is accurate then the lifetime in a pulsed mode could easily be extrapolated. 


\section{Plasma Source Energy Analyzer Experiment}

\subsection{Introduction}

A high-current density, high brightness ion beam is desired for Heavy Ion Fusion application. The intention is to achieve this by merging multiple lower current beams into a single high current beam. The plasma source test stand at LLNL is setup for proving this concept. The purpose of this experiment is to measure energy spread of the individual beamlets and the combined beam to demonstrate that while the effect of charge exchange with neutral gas is small and of little concern, it's effect can be seen and at the expected level.

During development of the source there initially was concern that collision of extracted ions with neutral gas in the diode gap would cause excessive charge exchange degrading the quality of the resulting beam. Gas atoms ionized in the gap would have inherently lower transverse energy and would contribute to an overall wider energy spread.

In order to accomplish this measurement a single-sector electrostatic spectrometer (energy analyzer) was used. The energy analyzer isolates from the beam, specific energy ions. Using the principle of electrostatic rigidity the instrument collects ions in a Faraday cup according to their energy. The curved electrode plates of the instrument produce a focusing effect to concentrate the mono-energetic beam on to a slit-cup detector. By varying the voltage on the electrodes, a scan of the beam's energy spread can be made. 


\subsection{Principles And Theory}

This section provides explanation for the principles used to conduct and explain the experimental results.

\subsubsection{Optics Of A Thin Lens}

The ability of the sector field to focus an incoming ion beam can be modeled using an optical lens model. The transformation of an image through an optical system can be described with transfer matrices. The final parameters can be calculated by knowing the initial coordinates and inclinations at the initial position. For an example the lens shown in figure 5.1 is used for a derivation of the transfer matrix.

$$
\left(\begin{array}{l}
x\left(z_{f}\right) \\
a\left(z_{f}\right)
\end{array}\right)=\left(\begin{array}{ll}
\left(x_{f} \mid x_{i}\right) & \left(x_{f} \mid a_{i}\right) \\
\left(a_{f} \mid x_{i}\right) & \left(a_{f} \mid a_{i}\right)
\end{array}\right)\left(\begin{array}{l}
x\left(z_{i}\right) \\
a\left(z_{i}\right)
\end{array}\right)
$$

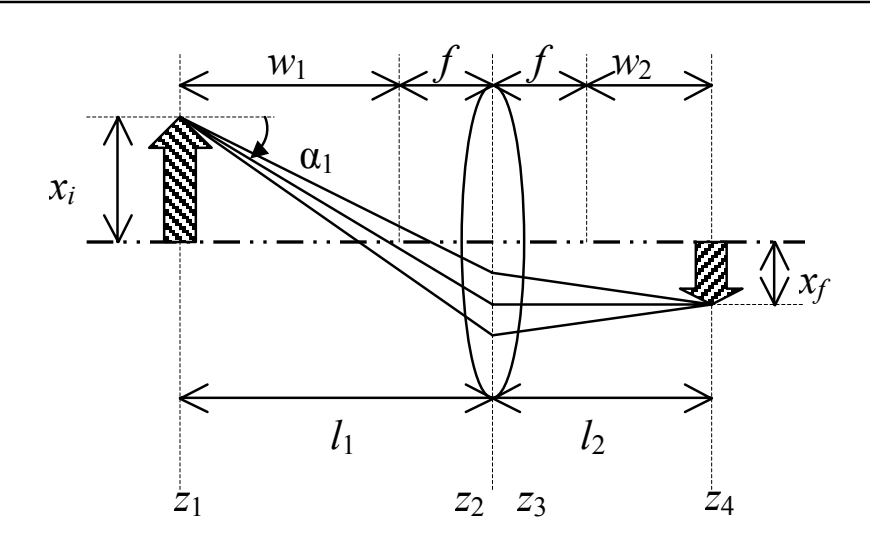

Figure 5.1 Thin lens with focal length $f$

For drift-length transfer ( $z_{1}$ to $z_{2}$ and $z_{3}$ to $\left.z_{4}\right)$ :

$$
T_{21}=\left[\begin{array}{cc}
1 & l_{1} \\
0 & 1
\end{array}\right] ; T_{43}=\left[\begin{array}{cc}
1 & l_{2} \\
0 & 1
\end{array}\right]
$$

For transfer through lens $\left(z_{2}\right.$ to $\left.z_{3}\right)$ :

$$
T_{32}=\left[\begin{array}{cc}
1 & 0 \\
-1 / f & 1
\end{array}\right]
$$


The entire transfer matrix for the system $\left(z_{1}\right.$ to $\left.z_{4}\right)$ :

$$
\begin{gathered}
{\left[\begin{array}{c}
x_{4} \\
\tan \alpha_{4}
\end{array}\right]=\left[\begin{array}{cc}
1 & l_{1} \\
0 & 1
\end{array}\right]\left[\begin{array}{cc}
1 & 0 \\
-1 / f & 1
\end{array}\right]\left[\begin{array}{cc}
1 & l_{2} \\
0 & 1
\end{array}\right]\left[\begin{array}{c}
x_{1} \\
\tan \alpha_{1}
\end{array}\right]} \\
=\left[\begin{array}{cc}
\left(1-l_{1} / f\right) & l_{1} \\
-1 / f & 1
\end{array}\right]\left[\begin{array}{cc}
1 & l_{2} \\
0 & 1
\end{array}\right]\left[\begin{array}{c}
x_{1} \\
\tan \alpha_{1}
\end{array}\right] \\
=\left[\begin{array}{cc}
\left(1-l_{1} / f\right) & \left(l_{2}-l_{1} l_{2} / f+l_{1}\right) \\
-1 / f & \left(-l_{2} / f+1\right)
\end{array}\right]\left[\begin{array}{c}
x_{1} \\
\tan \alpha_{1}
\end{array}\right]
\end{gathered}
$$

$l_{1}$ and $l_{2}$ are chosen so that the $(x \mid a)$ term is equal to zero giving:

$$
l_{1}+l_{2}-\left(\frac{l_{1} l_{2}}{f}\right)=0
$$

Which can be rearranged into the lens equation:

$$
\frac{1}{l_{1}}+\frac{1}{l_{2}}=\frac{1}{f}
$$

The maginfication of the lens is given by the $(x \mid x)$ term and for the thin lens is:

$$
M=(x \mid x)=1-\frac{l_{1}}{f}
$$

By the Liouville theorem the determinant of the transfer matrix is equal to one. This requires that $(x \mid x)(a \mid a)=1$ and produces the following :

$$
M=-\left(\frac{l_{2}}{l_{1}}\right)
$$

Then for the simple case where $l_{1}=l_{2}=f$, the magnification reduces to $M=-1$ and:

$$
\left[\begin{array}{c}
x_{4} \\
\tan \alpha_{4}
\end{array}\right]=\left[\begin{array}{cc}
0 & f \\
-1 / f & 0
\end{array}\right]\left[\begin{array}{c}
x_{1} \\
\tan \alpha_{1}
\end{array}\right]
$$




\subsubsection{Energy Analyzer Principles}

When charged a particle moves through the curved electrostatic field, for a relativistically slow particle, a simple relationship can be made by setting the centrifugal force equal to the centripetal Coulomb force.

$$
F_{\text {cenrrifigal }}=\frac{m v^{2}}{R} ; F_{\text {coulomb }}=q \vec{E}
$$

This gives the definition of Electrostatic Rigidity $\left(\chi_{e}\right)$ :

$$
\chi_{E}=E R=\frac{m v^{2}}{q}
$$

Where $R$ is the radius of beam path and $E$ is the electrostatic field. This further simplifies, for relativistically slow particles to:

$$
\chi_{E}=-2 T_{o}
$$

The electrostatic rigidity depends only on the accelerating potential $T_{\mathrm{o}}$, and not on the particle rest mass. Two particles of equal energy-to-charge ratios are deflected equally by the electrostatic field independent of the masses of the two particles. For the energy analyzer measuring $\leq 80 \mathrm{keV}$ ions, the non-relatavistic condition applies producing:

$$
\begin{gathered}
E=\frac{\Delta V}{d} \\
\Delta V=\frac{2 d}{R} T_{0}=(0.103) T_{0}
\end{gathered}
$$

The multiplication factor is a constant function of the instrument's geometry. The factor $(0.103)$ is used for all beam energies.

Transfer in the y-plane of the sector field can be described in a matrix similar to the previous thin lens example, but for the off axis, transfer will be simplified significantly. 


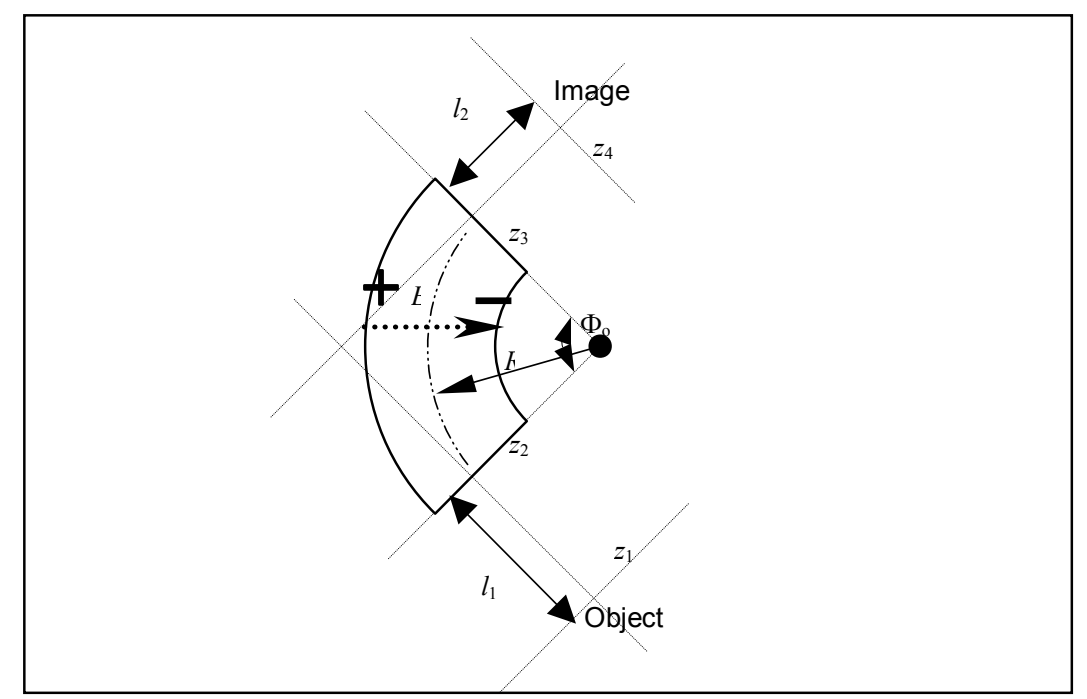

Figure 5.2. Geometry of optics.

The dimensions labeled in figure 5.2 are used for the following derivation. During drift lengths $l_{1}$ and $l_{2}$ the transfers ( $z_{1}$ to $z_{2}$ and $z_{3}$ to $\left.z_{4}\right)$ are simply:

$$
T_{21}=\left[\begin{array}{cc}
1 & l_{1} \\
0 & 1
\end{array}\right] ; T_{43}=\left[\begin{array}{cc}
1 & l_{2} \\
0 & 1
\end{array}\right]
$$

The transfer through the "lens" $\left(z_{2}\right.$ to $\left.z_{3}\right)$, in the plane of deflection, is a function of the angle of deflection $\phi_{0}$ and the radius of the bend $R$ :

$$
T_{32}=\left[\begin{array}{cc}
\cos \left(\sqrt{2} \phi_{0}\right) & \frac{R \sin \left(\sqrt{2} \phi_{0}\right)}{\sqrt{2}} \\
-\frac{\sqrt{2} \sin \left(\sqrt{2} \phi_{0}\right)}{R} & \cos \left(\sqrt{2} \phi_{0}\right)
\end{array}\right]
$$

The total system transfer in the plane of deflection is described by combining the three matrices:

$$
T_{x}=\left[\begin{array}{cc}
c_{x}-l_{2} S_{x} k_{x}^{2} & S_{x}+\left(l_{1}+l_{2}\right) c_{x}-l_{1} l_{2} S_{x} k_{x}^{2} \\
-s_{x} k_{x}^{2} & c_{x}-l_{1} S_{x} k_{x}^{2}
\end{array}\right]
$$

where:

$$
c_{x}=\cos \left(\sqrt{2} \phi_{0}\right), s_{x}=\frac{R}{\sqrt{2}} \sin \left(\sqrt{2} \phi_{0}\right), \text { and } k_{x}=\sqrt{2} / R \text { : }
$$


Particles continue unfocused in the plane perpendicular to the plane of deflection (yplane). Transfer in the y-plane can also be described in a matrix, however since no focusing occurs in the off axis, the transfer is consequently a simple drift-length transfer for the entire path length $l_{\mathrm{y}}=\left(l_{1}+R \phi_{0}+l_{2}\right)$ :

$$
T_{y}=\left[\begin{array}{ll}
1 & l_{y} \\
0 & 1
\end{array}\right]
$$

The instrument will be "in focus" when an object-image relationship to exists, which occurs when the $(x \mid a)$ term equals zero. Therefore, with $l_{1}$ fixed, the value for $l_{2}$ is found from:

$$
\begin{gathered}
(x \mid a)=s_{x}+\left(l_{1}+l_{2}\right) c_{x}-l_{1} l_{2} s_{x} k_{x}^{2}=0 \\
l_{2}=\frac{-l_{2} c_{x}-s_{x}}{c_{x}-l_{1} s_{x} k_{x}^{2}}=2.51^{\prime \prime}
\end{gathered}
$$

Magnification for the system is found from the $(x \mid x)$ term of the transfer matrix.

$$
M_{x}=(x \mid x)=c_{x}-l_{2} s_{x} k_{x}^{2}=-1.33
$$

In order to describe the resolution of the instrument the transfer matrix can be expanded to $3 \times 3$ to include a $\Delta$ term as in $R=R_{0}(1+\Delta)$.

$$
\left(\begin{array}{c}
x_{4} \\
a_{4} \\
\Delta
\end{array}\right)=\left(\begin{array}{ccc}
(x \mid x) & (x \mid a) & (x \mid \Delta) \\
(a \mid x) & (a \mid a) & (a \mid \Delta) \\
0 & 0 & 1
\end{array}\right)\left(\begin{array}{l}
x_{1} \\
a_{1} \\
\Delta
\end{array}\right)
$$

The rigidity dispersion $D_{\Delta}$ is defined as:

$$
D_{\Delta}=(x \mid \Delta)=d_{x}+\frac{l_{2} S_{x}}{R}=4.54 \quad\left\{d_{x}=\frac{1-\cos \left(\sqrt{2} \phi_{0}\right)}{R k_{x}^{2}}\right\}
$$

And the minimum resolvable $\Delta$ or the rigidity resolution:

$$
\Delta_{\min }=\frac{(x \mid x)}{(x \mid \Delta)} 2 w_{o}=\frac{M}{D_{\Delta}} 2 w_{o}=\frac{\left(2 w_{0}\right)\left(c_{x}-l_{2} S_{x} k_{x}{ }^{2}\right)}{d_{x}+\frac{l_{2} S_{x}}{R}}=5.29 \times 10^{-3}
$$




\subsubsection{Charge Exchange In The Diode Gap}

The plasma chamber is fed with an Argon gas by a periodic puff from a remote controlled valve. Because the diode is at vacuum and the chamber is at a positive pressure, the neutral gas will tend to leak from the chamber into the extraction gap. Neutral gas atoms in the gap provide a target for the ions as they move through the gap. These collisions, or ionizations, in the gap will contribute ions with lower kinetic energy to the beam. The concern was that this would present a significant problem for the overall beam quality.

To gain a better understanding of the problem the following model is constructed. The parameters are calculated and plotted as a function of transverse position $x$ in the diode gap $(1.6 \mathrm{~cm})$. Using the following relation, the expected effect on the output beam is predicted.

$$
I_{\Delta}=I_{o} \sigma n d x
$$

Where $I_{\Delta}$ represents the fractional change in initial beam current $I_{o}, \sigma$ is the crosssection for the ion to neutral collision, $n$ is the density of neutral gas atoms, and $d x$ the transverse distance traveled by the beam. For space-charge-limited flow the following equation describes the potential and the energy gained by the ions as they traverse the extraction gap. This is plotted in figure 5.3.

$$
E(x)=T_{o}\left[\frac{x}{1.6}\right]^{\frac{4}{3}}
$$




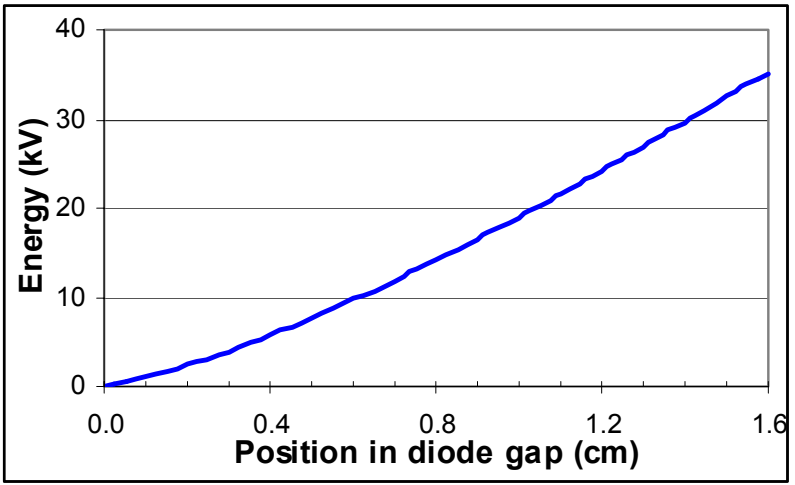

Figure 5.3. Energy of beam as it traverses the diode gap for $35 \mathrm{kV}$ extraction voltage.

Next the neutral gas density is found using a $1 / R^{2}$ model and the assumption that at room temperature, neutral atom density follows the ratio of $1 \mathrm{mT}=3.22 \times 10^{13} \mathrm{~cm}^{-3}$. This density is shown in figure 5.4, also as a function of position $x$.

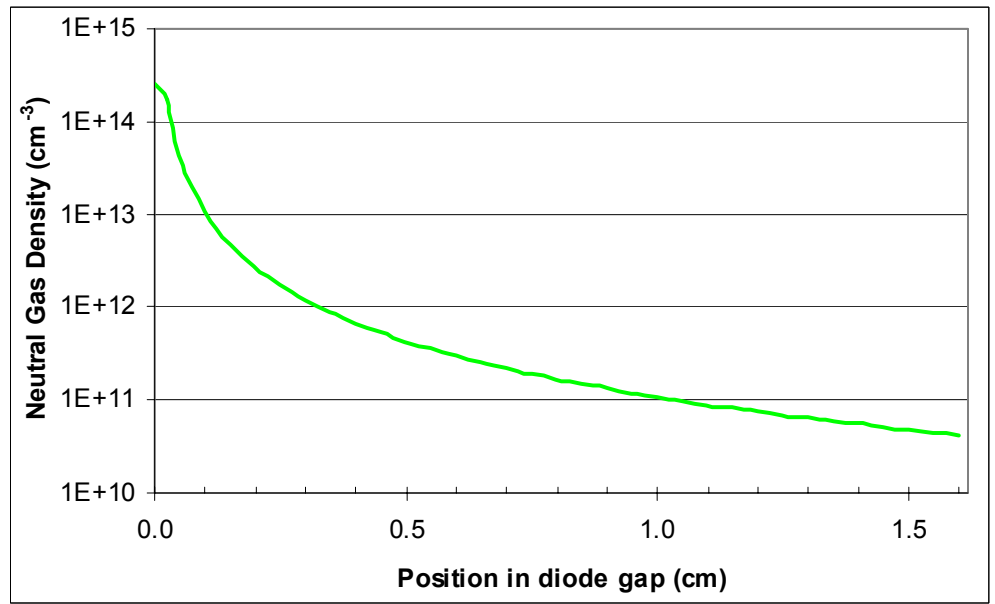

Figure 5.4. Model $\left(1 / R^{2}\right)$ used for neutral gas density in the diode gap using a $4 \mathrm{mT}$ initial pressure.

Next, with the use of the following equation, charge exchange cross-section for $\mathrm{Ar}^{+}$Ar is shown in figure 5.5.

$$
Q^{1 / 2} \times 10^{-8} \mathrm{~cm}^{2}=5.662-1.374 \log \left(E_{k e V}\right)
$$




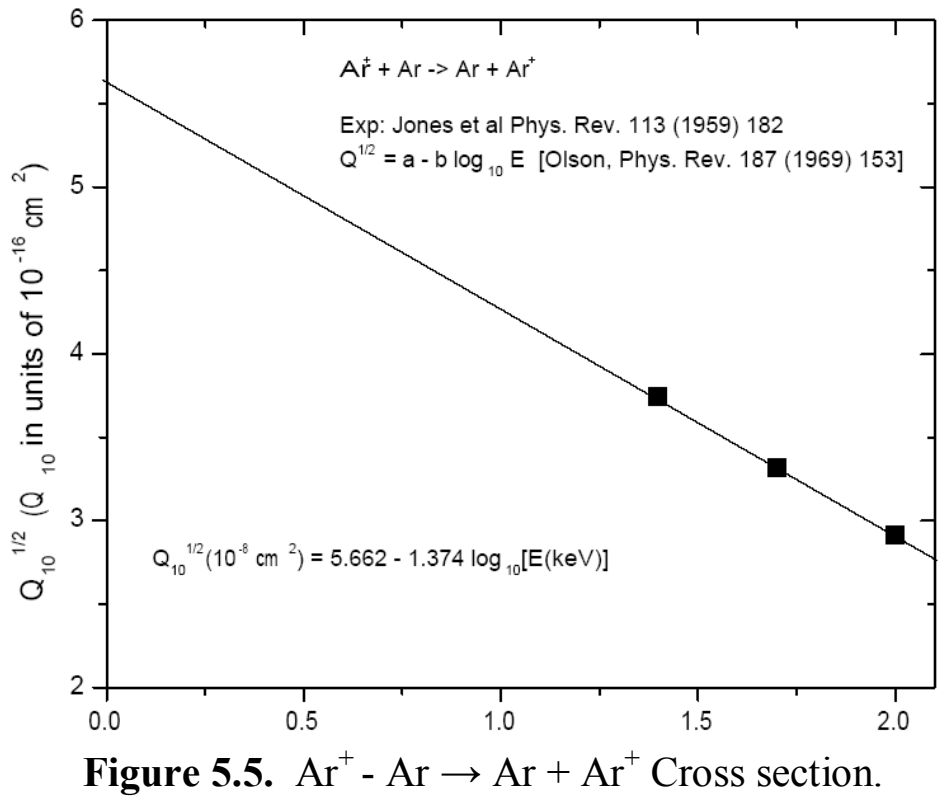

Then by combining the expressions for $E$ and $Q$ we obtain a charge exchange crosssection as a function of position in the gap shown in figure 5.6:

$$
\sigma(x)=Q(E(x))
$$

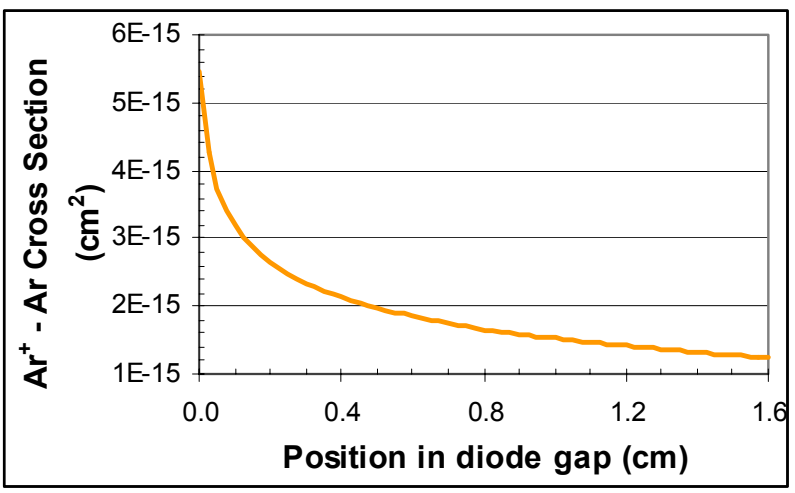

Figure 5.6. Charge exchange cross-section plotted as a function of position in diode gap.

The final result are the curves of figure 5.7. These show the expected energy spread of a $35 \mathrm{kV} \mathrm{Ar}^{+}$beam with respect to neutral Ar gas pressure as a fraction of beam current. The experimental results section below will compare this model to the actual measured data. 


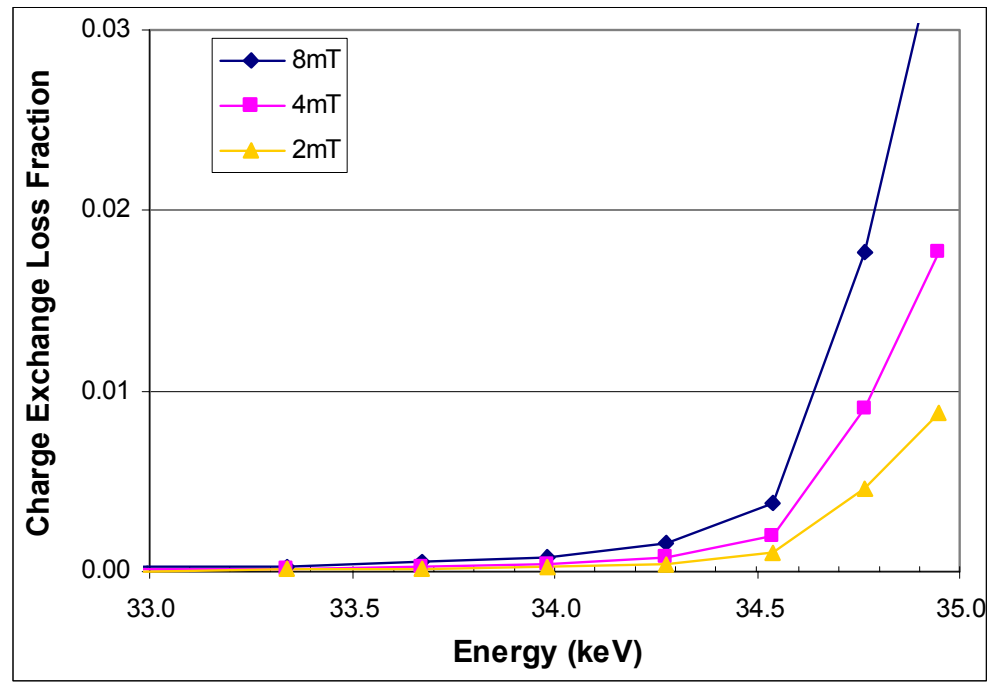

Figure 5.7. Fractional loss due to charge exchange for varied neutral gas pressures.

\subsection{Experiment Setup}

\subsubsection{Source Setup}

The ion source test stand used for this experiment (STS 100 at LLNL) operates with an RF driven plasma source. The source is configured with a $26 \mathrm{~cm}$ inner diameter cylindrical plasma chamber, multi-cusp permanent magnet confinement, $11 \mathrm{MHz}$ / $20 \mu \mathrm{s}$ RF pulse, through a 2 turn $11 \mathrm{~cm}$ antenna. Ar gas is fed through a time controlled puffer valve. The multi-beam array consists of a 61 hole grid in a hexagonal pattern. The beam forming plate contains $2.2 \mathrm{~mm}$ Pierce cones, the extraction plate has matching $4.0 \mathrm{~mm}$ holes and the gap between the plates is $1.6 \mathrm{~cm}$, capable of holding $80 \mathrm{kV}$.

\subsubsection{Energy Analyzer Setup}

The energy analyzer is shown in Figure 5.8 with the basic configuration and the significant dimensions of the setup. DC voltages are applied to each curved plate electrodes; one positive and one negative producing a GND potential at the centerline of curved beam path. Three mechanical parameters of the instrument are adjustable: Focus $\left(l_{l}\right)$, Faraday cup cant, and overall up-down position of instrument with respect to source. 

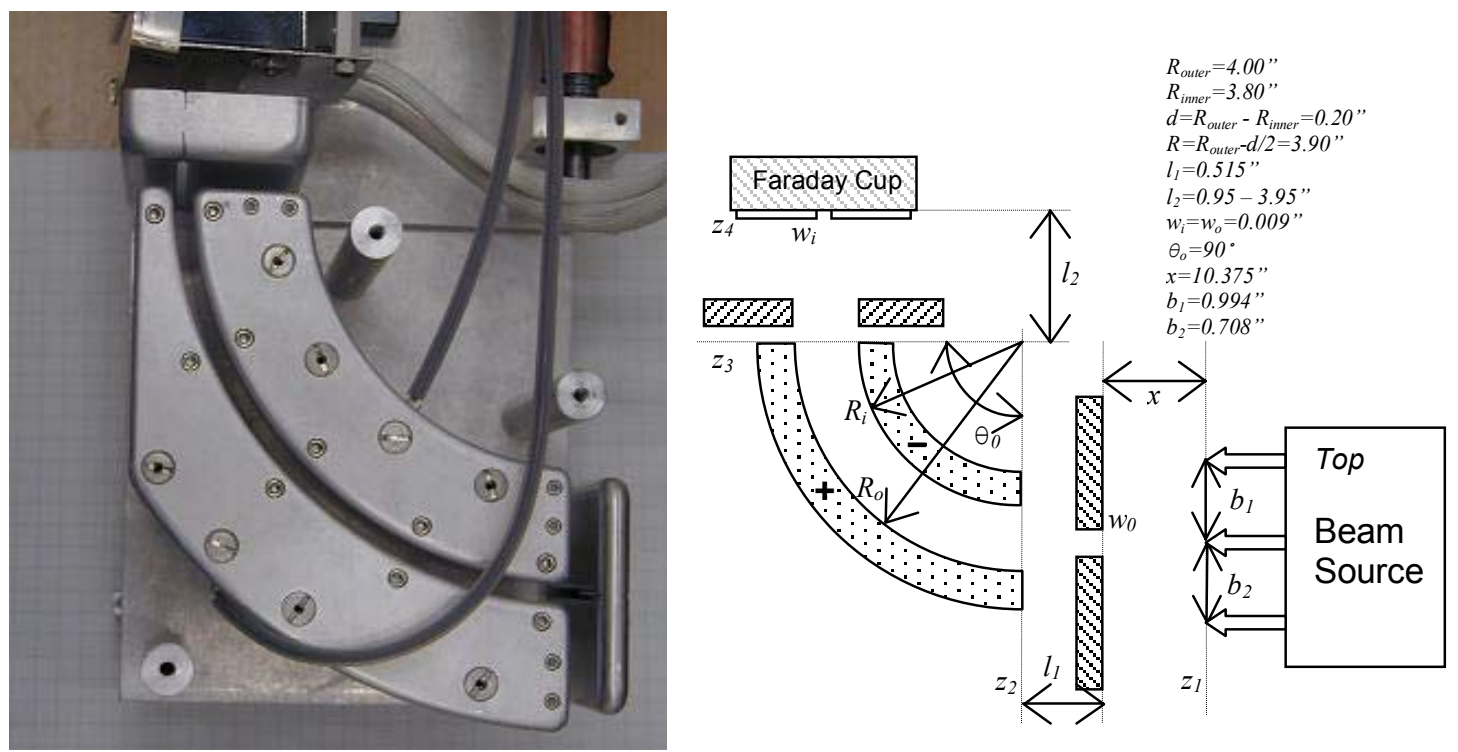

Figure 5.8. Energy analyzer and schematic.

The electrodes are connected to their respective power supplies through coupling boxes shown in figure 5.9. This circuit is designed to mitigate the voltage drop due to beam striking the analyzer electrodes. Additionally, an output is provided to allow monitoring signal. During initial runs the pulse appeared to move erratically in time. After diagnosis of the electrode signals, a second capacitor (50nF) was added, due to a stray signal on the negative (closer to plasma chamber) electrode. The signal was believed to be caused by the close proximity to either the plasma or the RF system. After addition of the second capacitor, the pulse was very steady. Calibration of electrode power supplies was conducted using a precision high-voltage divider, with measurements take on a Hewlett Packard high accuracy DVM. 


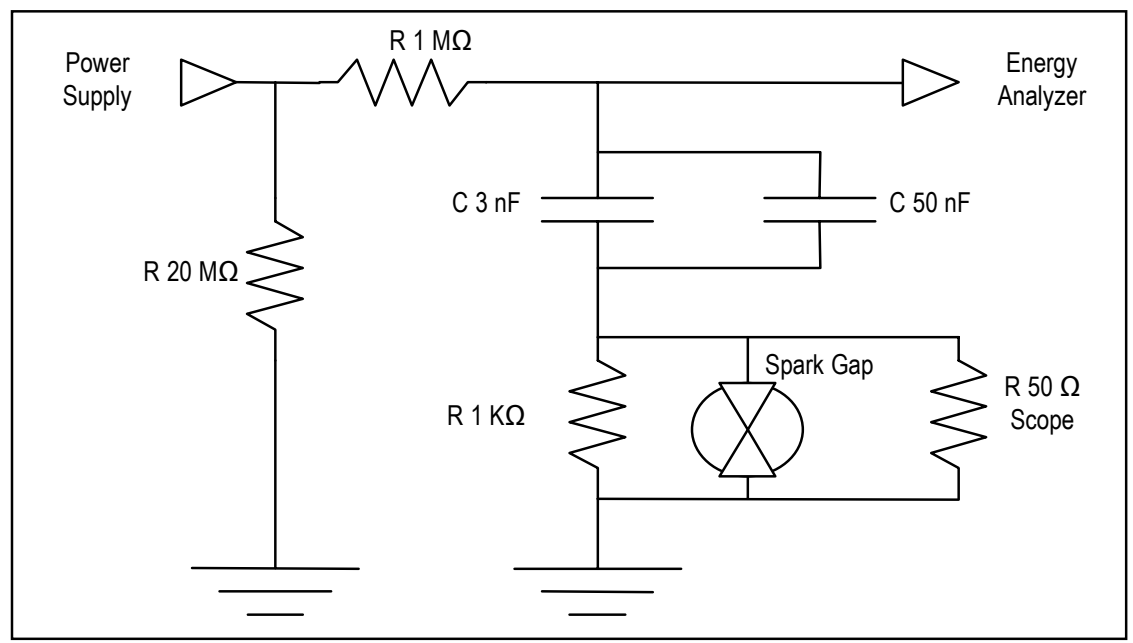

Figure 5.9. Coupling Box Circuits (+ and -)

\subsubsection{High Voltage Pulse}

During the single beamlet tests, the extraction voltage was set at $45 \mathrm{kV}$ and the pulse droop was approximately $27.48 \mathrm{~V} / \mu$ s as shown in figure 5.10 . This droop causes an overall voltage change of about $824.4 \mathrm{~V}$ over the $30 \mu$ s duration. Consequently a voltage scan is produced without requiring adjustment of the energy analyzer electrode voltages.

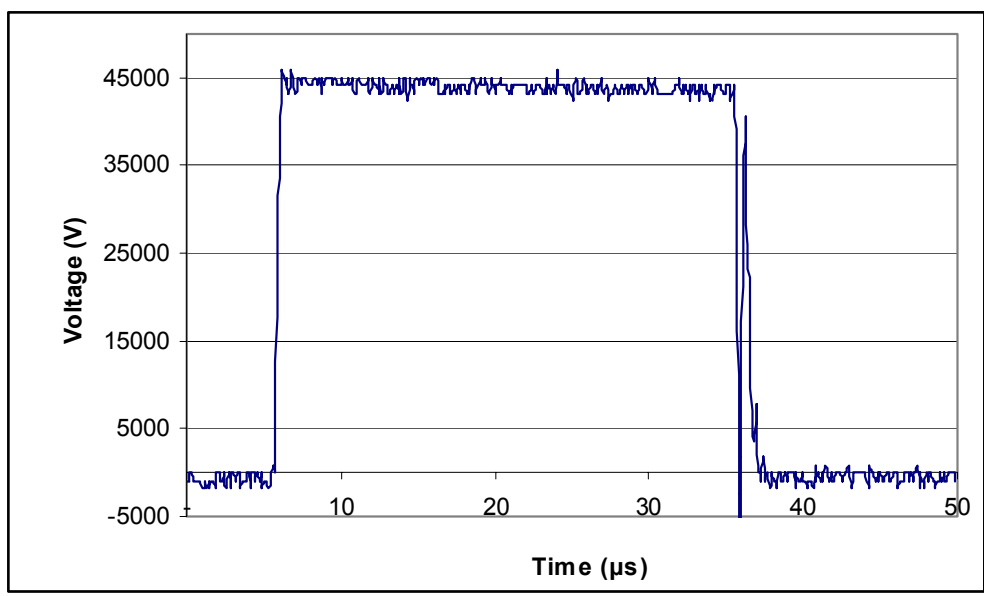

Figure 5.10. $45 \mathrm{kV}$ extraction pulse, droop is $27.48 \mathrm{~V} / \mu \mathrm{s}$.

For the combined beam tests, the extraction pulse length was increased, and the following figure 5.11 shows the droop associated with the two settings used. 


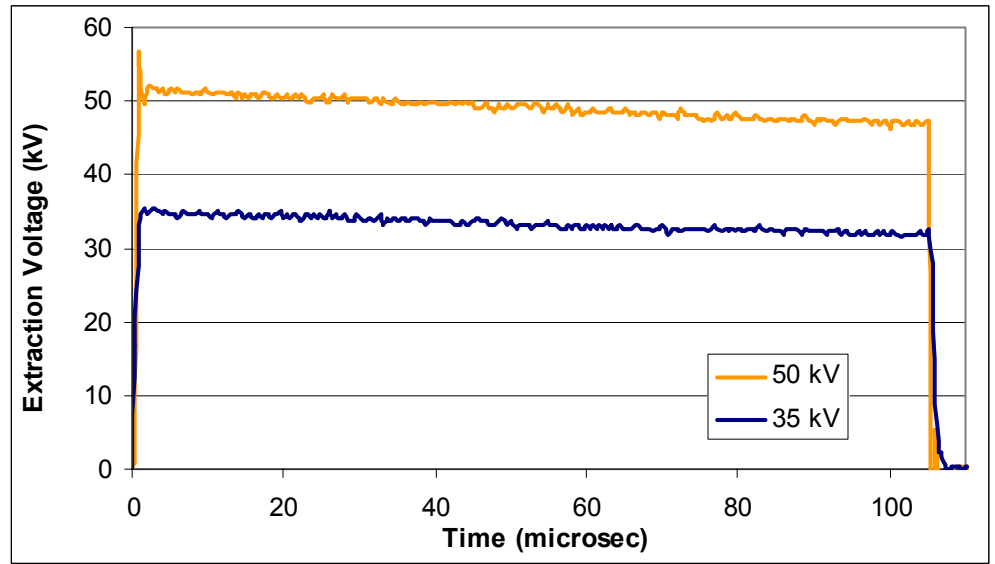

Figure 5.11. High voltage droop, $35 \mathrm{kV}-29.66 \mathrm{~V} / \mu \mathrm{s}, 50 \mathrm{kV}-46.84 \mathrm{~V} / \mu \mathrm{s}$.

\subsection{Single Beamlet Results}

Figure 5.12 shows a resultant signal generated in the analyzer Faraday cup with electrode voltages held constant. The pulse width is approximately $9 \mu$ s which corresponds to $247.32 \mathrm{~V}$ change in beam energy.

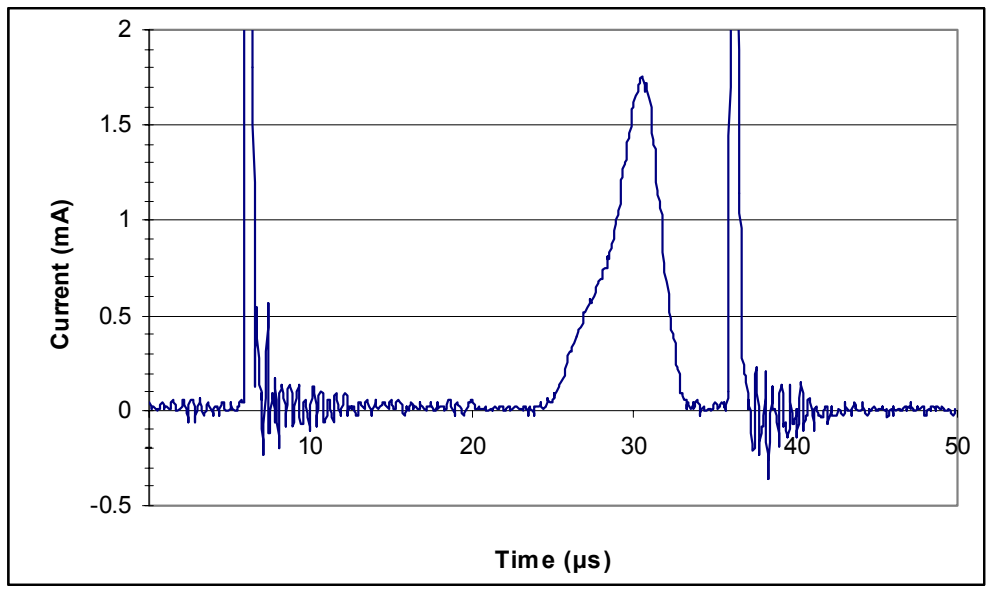

Figure 5.12. Energy Analyzer Signal (45kV, center beamlet).

\subsubsection{Adjustments To Instrument}

The focus $\left(l_{2}\right)$ was adjusted over the full range of motion and a sample of the result can be seen in figure 5.13. The shape shifts and the peak changes, however the overall width of the pulse remains relatively constant up to 2.56 " and increases only slightly all the way in to 0.95 ". By calculations the focal point should have been at 2.51 ", and while 
the width does increase after this point, the peak also continues to rise as the cup is moved in past this point.

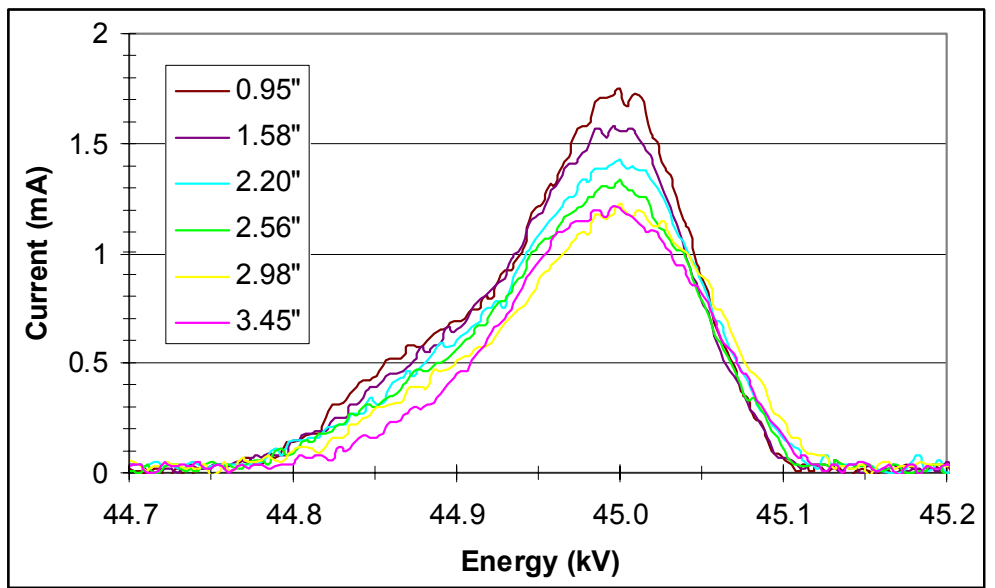

Figure 5.13. Various focus positions on center beamlet $(45 \mathrm{kV})$

The instrument provides the ability to adjust the cant angle of the Faraday cup with respect to the outlet of the electrodes. This was adjusted to achieve the highest amplitude signal, and once found; the setting was left constant for all experiments.

Stage position adjustment was used to switch between upper and middle beams. With all parameters left constant, a significant difference exists between the upper and middle beams, and can be seen in figure 5.14 .

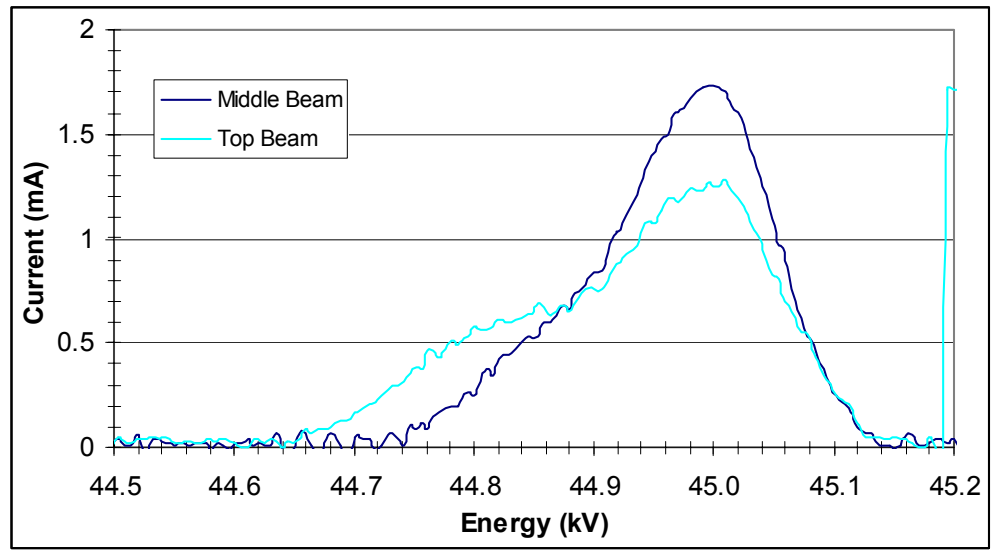

Figure 5.14. Top vs. Middle Beam (45kV). 


\subsubsection{Argon Gas Pressure Variations And Charge Exchange For Single Beamlet}

Gas pressure was varied over a wide range to explore the presence of charge exchange effects. As can be seen from figure 5.15, the width of the pulse appears to have remained constant, indicating that the measurable beam energy spread was unaffected. The overall amplitude however was noticeably decreased at higher pressures.

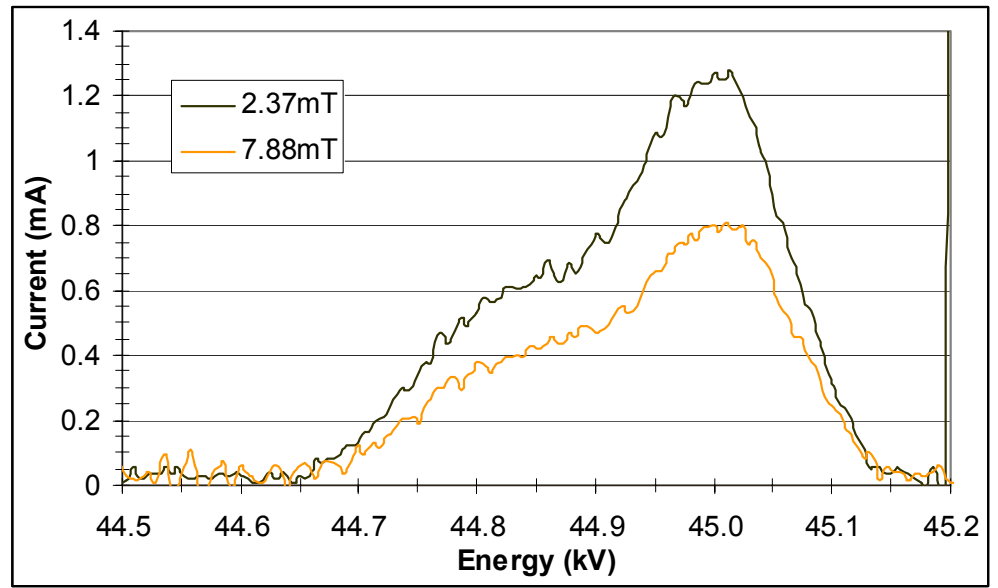

Figure 5.15. Effect of gas pressure variations $(45 \mathrm{kV}$, center beam).

Gas pressure causes the shape of the source meniscus to change; this affects the beam optics such that less beam current strikes the analyzer entrance slit due to greater divergence resulting in the lower signal amplitude. The overall energy spread however appears to be unaffected, which is contrary to what is expected. The effect of charge exchange due to neutral gas in the diode is in fact small and is shown below in section 4.3 .

\subsubsection{Scanning Of Electrode-Voltage}

For scanning data runs, the beam voltage was held constant while the electrode voltage was adjusted before each shot by a preset delta. Figure 5.16 shows a 3D representation of $39-0.02 \mathrm{kV}$ steps plotted on the same axis. As the electrode voltages increased, the pulse slid earlier in time than the previous pulse. This effect is caused by 
the high-voltage pulse droop and the diagonal line corresponds to the slope of this droop. For this example beam energy was $50 \mathrm{kV}$ and the slope calculated from the graph is:

$$
m=\frac{\Delta V}{\Delta t}=\frac{50.22-49.58}{16}=40 \mathrm{~V} / \mu \mathrm{s}
$$

Figure 5.17 is a graph of the HV pulse used to produce figure 5.16, and the slope matches the above calculation of $40 \mathrm{~V} / \mu \mathrm{s}$.

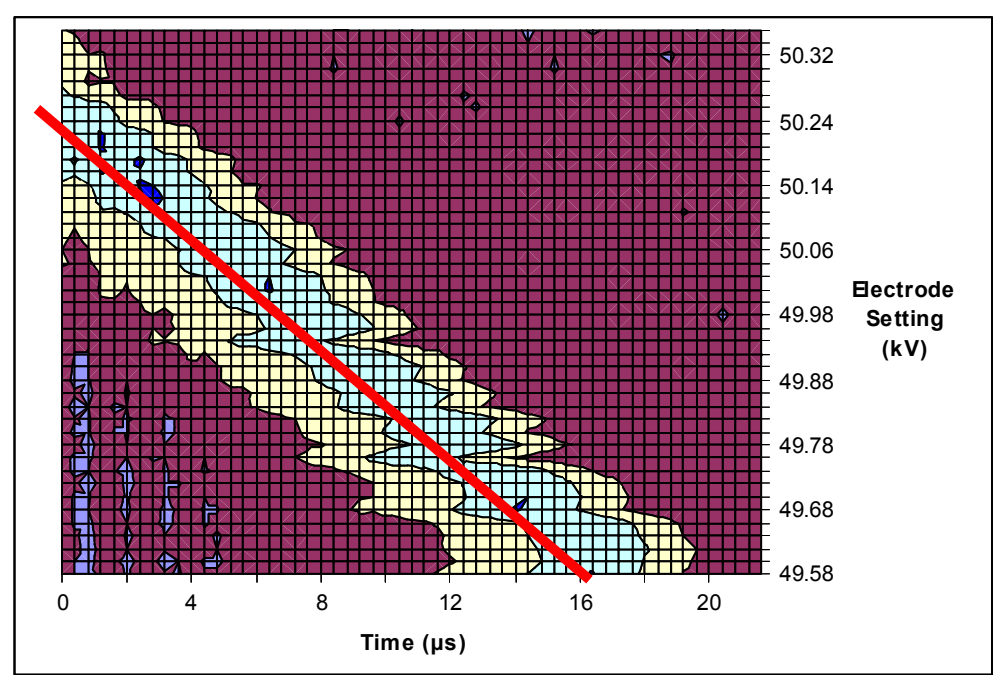

Figure 5.16. Electrode voltage scan showing pulse droop $(50 \mathrm{kV})$

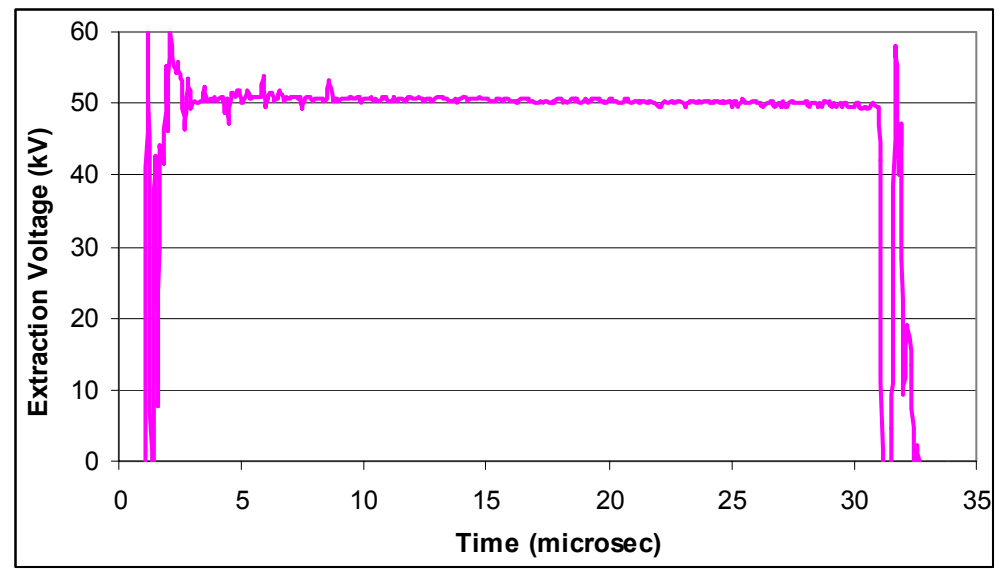

Figure 5.17. High voltage droop for the $50 \mathrm{kV}$ pulse used to generate figure 15 . The slope for this case is $40 \mathrm{~V} / \mu \mathrm{s}$. 


\subsubsection{Asymmetric Electrode-Voltage Experiment}

In an attempt to calibrate the instrument versus beam energy the electrodes were set asymmetrically to test the effect on the results. With $45 \mathrm{kV}$ extraction voltage, electrode voltages are set at $\pm 2.298 \mathrm{kV}(\Delta \mathrm{V}$ of $4.596 \mathrm{kV})$ to center the signal, this corresponds to $44.8 \mathrm{kV}$ beam energy. The plate voltages were then set asymmetrically by placing the negative electrode at ground and adjusting the positive electrode until the pulse was again centered. A positive $4.391 \mathrm{kV}$ was required to center the pulse therefore creating a center voltage of $2.196 \mathrm{kV}$. The $\Delta \mathrm{V}$ of $4.391 \mathrm{kV}$ corresponds to $42.8 \mathrm{kV}$ beam energy. The asymmetric setup introduces a deceleration caused by the positive centerline voltage and is approximately $(\sim \% 10)$ equal to the $2.0 \mathrm{kV}$ difference seen in beam energy.

$$
\begin{aligned}
& T=k \Delta V \\
& \delta T=2.196 \\
& \delta \Delta V=2(2.298)-4.391=0.205 \\
& k=\frac{2.196}{0.205}=10.712 \\
& \therefore T=10.712(2)(2.298)=49.232 \mathrm{kV} \\
& \Delta \%=\frac{49.232-45}{45}=9.40 \%
\end{aligned}
$$

\subsection{Combined Beam Results}

As shown in section 2.1 the ideal minimum resolution of the energy analyzer is approximately $0.5 \%$. Comparing the signals seen in the figures 5.18 and 5.19 , given the accuracy of the instrument, it appears the actual energy spread of beam is being measured. 


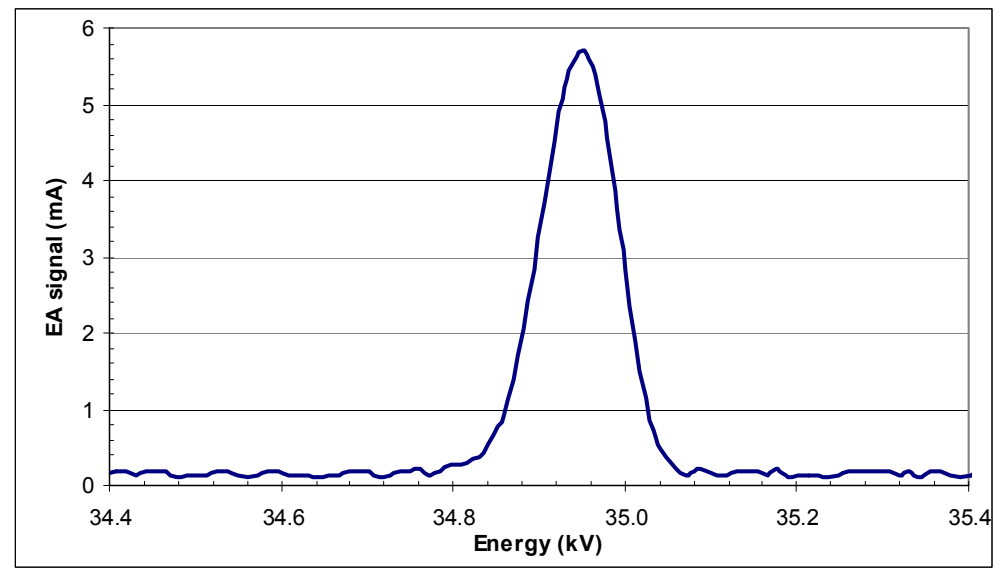

Figure 5.18. Energy analyzer signal for $35 \mathrm{kV}$ beam using $4 \mathrm{~ms}$ on gas valve. Total energy spread is approx $240 \mathrm{~V}$ and peak value of the signal is $5.6 \mathrm{~mA}$.

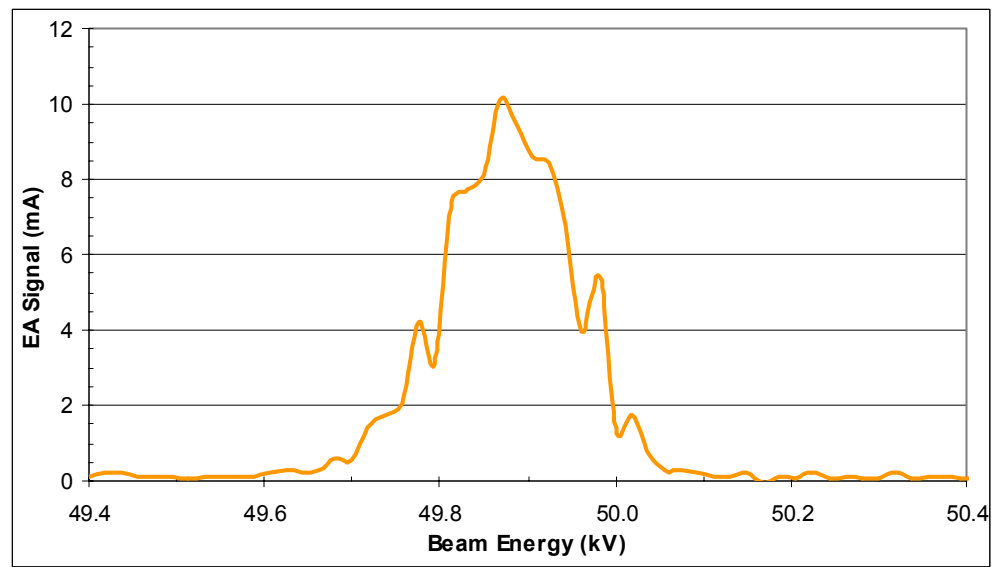

Figure 5.19. Energy analyzer signal for $50 \mathrm{kV}$ beam using $4 \mathrm{~ms}$ on gas valve. Total energy spread is approx $400 \mathrm{~V}$ and peak value of the signal is $10.0 \mathrm{~mA}$.

The peak current values nearly track with the $\Phi^{3 / 2}$ law as follows:

$$
\begin{gathered}
J \propto \Phi^{3 / 2} \\
\frac{J_{35 k V}}{J_{50 k V}}=0.570 \approx\left(\frac{35 k V}{50 k V}\right)^{3 / 2}=0.585
\end{gathered}
$$

\begin{tabular}{|c|c|c|c|}
\hline $\begin{array}{c}\text { Beam Energy } \\
(\mathrm{kV})\end{array}$ & $\begin{array}{c}\text { Min Analyzer } \\
\text { Resolution (V) }\end{array}$ & $\begin{array}{c}\text { Energy } \\
\text { Spread (V) }\end{array}$ & $\begin{array}{c}\text { Energy spread } \\
\text { percentage }\end{array}$ \\
\hline 35 & 186 & 240 & $0.7 \%$ \\
\hline 50 & 265 & 400 & $0.8 \%$ \\
\hline
\end{tabular}

Table 5.1. Values for results shown in figures 5.18 and 5.19. 


\subsection{Energy Spread Due To Charge Exchange}

From figure 5.20 the effect of charge exchange with the neutral gas can be seen increasing with higher gas pressures.

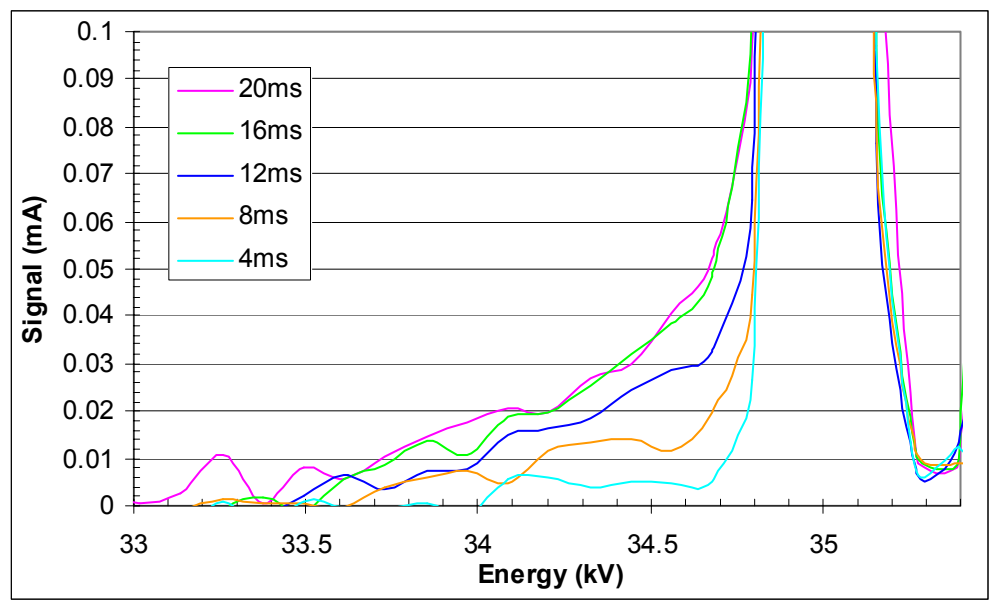

Figure 5.20. Measured signal for five different gas pressures with $35 \mathrm{kV}$ extraction.

Comparing figure 5.20 to 5.21 , it can be seen that higher extraction voltage will increase the effect proportionally with the higher beam current.

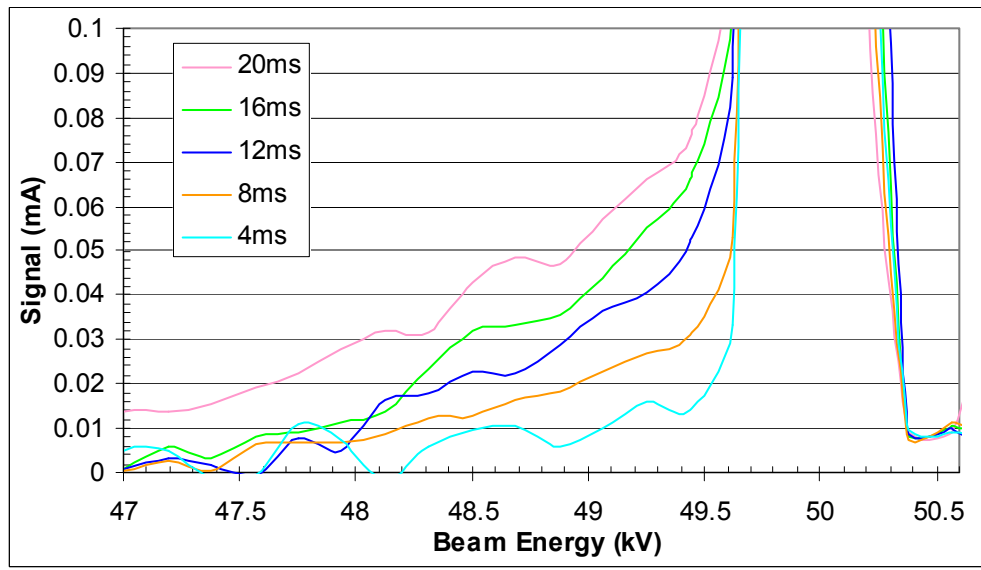

Figure 5.21. Measured signal for five different gas pressures with $50 \mathrm{kV}$ extraction.

The measured signals are smoothed as shown in figure 5.22 to generate a base fit, and then as shown the signal for each gas pressure is a multiple of the base fit. Therefore the following calculations are more easily conducted from this base curve. 


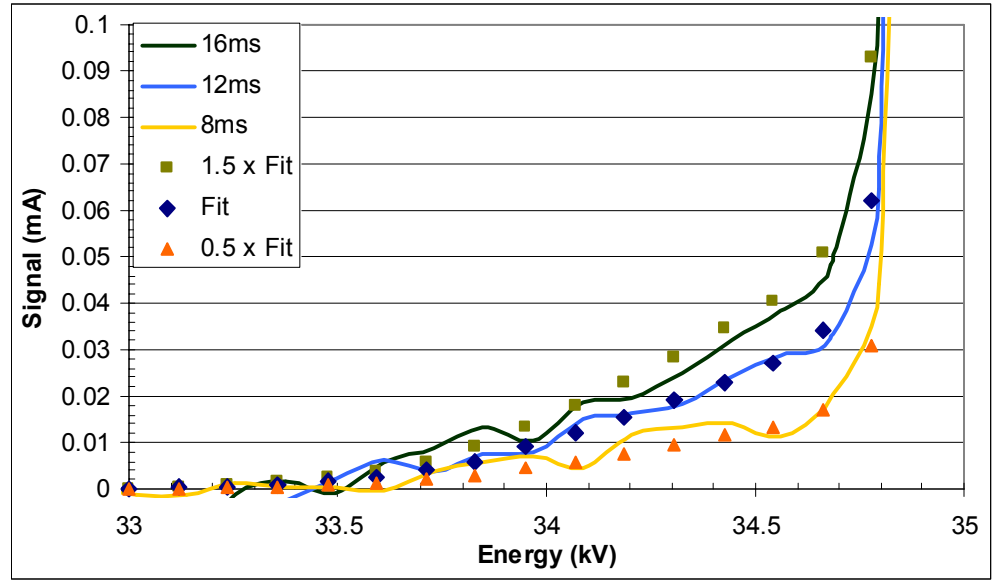

Figure 5.22. Smooth curves fitted to the $35 \mathrm{kV}$ measured signal. The curves above and below the fit are simply multiples of the base fit showing how gas pressure is proportional to measured signal.

The dashed line in figure 5.23 shows the value as predicted using $1 / R^{2}$ gas density model. The important factor here is to demonstrate that the approximate magnitude of energy spread due to charge exchange is in the predictable range. The difference between the actual signal and calculated model is most likely due to an inaccurate model for the neutral gas density in the gap.

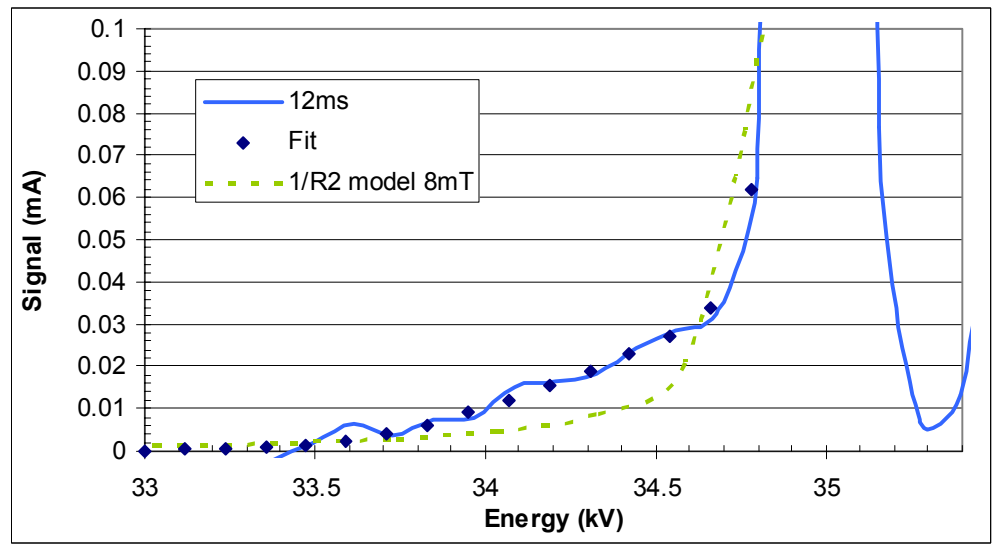

Figure 5.23. Actual signal compared to fitted curve and predicted curve using a $1 / R^{2}$ gas density model. 
In order to develop a better fit between the model and actual measured signal, gas density is found by starting with the measured signal and using the same method in reverse shown in figure 5.24.

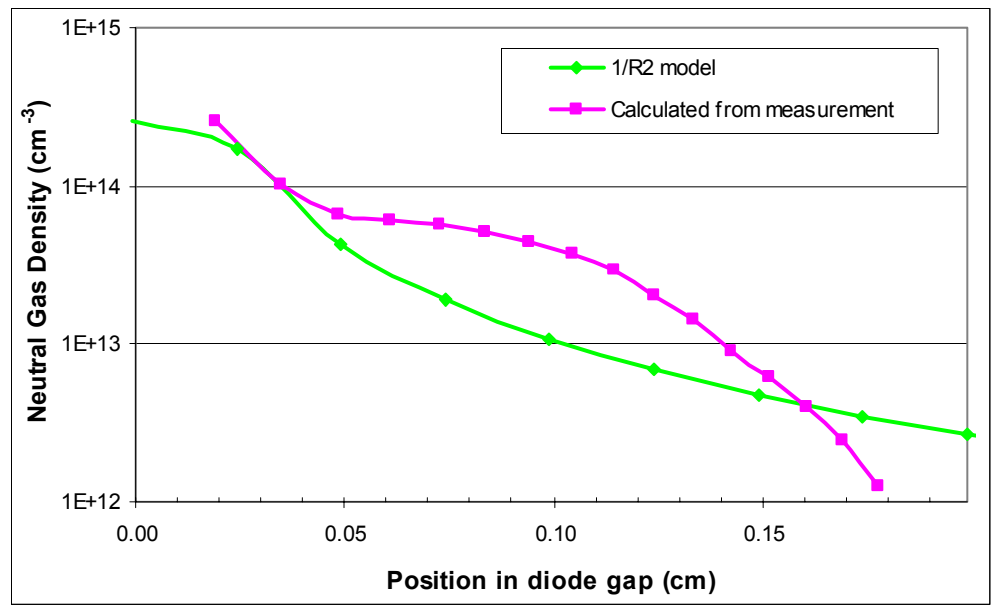

Figure 5.24. Calculated (from measured signal) gas density shown versus $1 / \mathrm{R}^{2}$ model gas density. Gas density axis in this graph is shown in log scale.

\subsection{Conclusions}

The energy spread of the plasma source beam is less than $1 \%$ for these extraction voltages, and the component resulting from charge exchange is in fact much less than initially expected. Consequently, charge exchange does not present a significant problem for the overall beam quality. A useful product of this set of experiments is a neutral gas density profile for the multi-beam grid. 


\section{References and Acknowledgements}

Baca, D., et al. Fabrication of Large Diameter Alumio-Silicate K Sources. Proceedings of the Particle Accelerator Conference. 2003.

Chacon-Glocher, E., Studies in High Current Density Ion Sources for Heavy Ion Fusion Applications. Ph.D. Dissertation, University of California at Berkeley, 2002.

Forrester, T.A., Large Ion Beams, Fundamentals of Generation and Propagation. John Wiley \& Sons, 1978.

Humphries, S. Jr., Charged Particle Beams. John Wiley \& Sons, 1990.

Kwan, J.W., et al. Development of High Current Density Surface Ionization Sources for Heavy Ion Fusion. Proceedings of the Particle Accelerator Conference. 2001.

Westenskow, G.A., et al. High Current Ion Source Development for Heavy Ion Fusion. 2003.

Wollnik, H., Optics of Charged Particles. Academic Press, 1987

I would like to thank the following for their invaluable supervision, guidance and assistance:

LBNL: Dr. Joe Kwan, David Baca, Will Waldron

LLNL: Dr. Glen Westenskow, Robert Hall, Gary Freeze

UCB: Prof. Per Peterson, Prof. Ka-Ngo Leung

Additionally, I am grateful to the Lawrence Berkeley National Laboratory for financial support and use of equipment and facilities making this all possible. 